\title{
Program Algebra over an Algebra ${ }^{1}$
}

\author{
Grzegorz Bancerek \\ Faculty of Computer Science \\ Białystok Technical University \\ Wiejska 45A, 15-351 Białystok, Poland
}

\begin{abstract}
Summary. We introduce an algebra with free variables, an algebra with undefined values, a program algebra over a term algebra, an algebra with integers, and an algebra with arrays. Program algebra is defined as universal algebra with assignments. Programs depend on the set of generators with supporting variables and supporting terms which determine the value of free variables in the next state. The execution of a program is changing state according to successor function using supporting terms.
\end{abstract}

MML identifier: AOFA_A00, version: $\underline{8.0 .01 \quad 5.3 .1165}$

The terminology and notation used in this paper have been introduced in the following papers: [40], [9], [24], [16], [25], [1], [3], [7], [28], [13], [32], [10], [12], [17], [38], [23], [31], [18], [19], [20], [5], [14], [8], [37], [41], [36], [30], [35], [11], [34], [26], [4], [21], [33], [29], [42], [39], [2], [6], [27], [15], and [22].

\section{Preliminaries}

For simplicity, we adopt the following convention: $i$ denotes a natural number, $x, y, z$ denote sets, $\Sigma$ denotes a non empty non void many sorted signature, and $X$ denotes a non-empty many sorted set indexed by the carrier of $\Sigma$.

We now state three propositions:

(1) For all sets $A, B$ and for every $A$-valued binary relation $R$ holds $R^{\circ} B \subseteq A$.

\footnotetext{
${ }^{1}$ This work has been supported by the Polish Ministry of Science and Higher Education project "Managing a Large Repository of Computer-verified Mathematical Knowledge" (N N519 385136).

(C) 2012 University of Białystok CC-BY-SA License ver. 3.0 or late ISSN 1426-2630(p), 1898-9934(e)
} 
(2) For all sets $I, J$ such that $I \subseteq J$ holds $I^{i} \subseteq J^{i}$.

(3) Let $I, J$ be non empty sets and $f$ be a homogeneous partial function from $I^{*}$ to $J$. Then $f$ is quasi total and non empty if and only if $\operatorname{dom} f=I^{\operatorname{arity} f}$.

Let $I$ be a set, let $f$ be a many sorted set indexed by $I$, let $i$ be a set, and let us consider $x$. Then $f+\cdot(i, x)$ is a many sorted set indexed by $I$.

Let $A, B$ be sets, let $f$ be a function from $A$ into $B$, let $x$ be a set, and let $y$ be an element of $B$. Then $f+\cdot(x, y)$ is a function from $A$ into $B$.

Let $I$ be a set, let $A, B$ be many sorted sets indexed by $I$, let $F$ be a many sorted function from $A$ into $B$, and let us consider $x$. Then $F(x)$ is a function from $A(x)$ into $B(x)$.

Let $I$ be a set, let $f$ be a non-empty many sorted set indexed by $I$, let $i$ be a set, and let $x$ be a non empty set. Note that $f+\cdot(i, x)$ is non-empty.

The following propositions are true:

(4) For every set $I$ and for all many sorted sets $f, g$ indexed by $I$ such that $f \subseteq g$ holds $f^{\#} \subseteq g^{\#}$.

(5) Let $I$ be a non empty set, $J$ be a set, and $A, B$ be many sorted sets indexed by $I$. If $A \subseteq B$, then for every function $f$ from $J$ into $I$ holds $A \cdot f \subseteq B \cdot f$.

(6) For every set $I$ and for all many sorted sets $A, B$ indexed by $I$ such that $A \subseteq B$ holds $\prod A \subseteq \prod B$.

Let $f$ be a function yielding function. Note that Frege $(f)$ is function yielding.

The following two propositions are true:

(7) For all function yielding functions $f, g$ holds $\operatorname{dom}_{\kappa}(f \cdot g)(\kappa)=$ $\left(\operatorname{dom}_{\kappa} f(\kappa)\right) \cdot g$.

(8) For all functions $f, g$ such that $g=f(x)$ holds $g(y)=f(x)(y)$.

Let $I$ be a set, let $i$ be an element of $I$, and let us consider $x$. The functor $i$-singleton $x$ yields a many sorted set indexed by $I$ and is defined by:

(Def. 1) $i$-singleton $x=\mathbf{0 .} I+\cdot(i,\{x\})$.

One can prove the following propositions:

(9) For every non empty set $I$ and for all elements $i, j$ of $I$ and for every $x$ holds $(i$-singleton $x)(i)=\{x\}$ and if $i \neq j$, then $(i$-singleton $x)(j)=\emptyset$.

(10) Let $I$ be a non empty set, $i$ be an element of $I, A$ be a many sorted set indexed by $I$, and given $x$. If $x \in A(i)$, then $i$-singleton $x$ is a many sorted subset of $A$.

Let $I$ be a set, let $A, B$ be many sorted sets indexed by $I$, let $F$ be a many sorted function from $A$ into $B$, and let $i$ be a set. Let us assume that $i \in I$. Let $j$ be a set. Let us assume that $j \in A(i)$. Let $v$ be a set. Let us assume that $v \in B(i)$. The functor $F+\cdot(i, j, v)$ yields a many sorted function from $A$ into $B$ and is defined as follows: 
(Def. 2) $\quad(F+\cdot(i, j, v))(i)=F(i)+\cdot(j, v)$ and for every set $s$ such that $s \in I$ and $s \neq i$ holds $(F+\cdot(i, j, v))(s)=F(s)$.

Let $a, b, c, d, x, y, z, v$ be sets. The functor $(a, b, c, d) \mapsto(x, y, z, v)$ yielding a set is defined as follows:

(Def. 3) $(a, b, c, d) \mapsto(x, y, z, v)=(a, b, c) \mapsto(x, y, z)+\cdot(d \longmapsto v)$.

Let $a, b, c, d, x, y, z, v$ be sets. Observe that $(a, b, c, d) \mapsto(x, y, z, v)$ is relation-like and function-like.

Next we state a number of propositions:

(11) Let $a_{1}, a_{2}, a_{3}, b_{1}, b_{2}, b_{3}$ be sets. Then $\left(\left(a_{1}, a_{2}, a_{3}\right) \mapsto\left(b_{1}, b_{2}, b_{3}\right)\right)\left(a_{3}\right)=b_{3}$ and if $a_{2} \neq a_{3}$, then $\left(\left(a_{1}, a_{2}, a_{3}\right) \mapsto\left(b_{1}, b_{2}, b_{3}\right)\right)\left(a_{2}\right)=b_{2}$ and if $a_{1} \neq a_{2}$ and $a_{1} \neq a_{3}$, then $\left(\left(a_{1}, a_{2}, a_{3}\right) \mapsto\left(b_{1}, b_{2}, b_{3}\right)\right)\left(a_{1}\right)=b_{1}$.

(12) For all sets $a_{1}, a_{2}, a_{3}, a_{4}, b_{1}, b_{2}, b_{3}, b_{4}$ holds $\operatorname{dom}\left(\left(a_{1}, a_{2}, a_{3}, a_{4}\right) \mapsto\right.$ $\left.\left(b_{1}, b_{2}, b_{3}, b_{4}\right)\right)=\left\{a_{1}, a_{2}, a_{3}, a_{4}\right\}$.

(13) Let $a_{1}, a_{2}, a_{3}, a_{4}, b_{1}, b_{2}, b_{3}, b_{4}$ be sets. Then

(i) $\quad\left(\left(a_{1}, a_{2}, a_{3}, a_{4}\right) \mapsto\left(b_{1}, b_{2}, b_{3}, b_{4}\right)\right)\left(a_{4}\right)=b_{4}$,

(ii) if $a_{3} \neq a_{4}$, then $\left(\left(a_{1}, a_{2}, a_{3}, a_{4}\right) \mapsto\left(b_{1}, b_{2}, b_{3}, b_{4}\right)\right)\left(a_{3}\right)=b_{3}$,

(iii) if $a_{2} \neq a_{3}$ and $a_{2} \neq a_{4}$, then $\left(\left(a_{1}, a_{2}, a_{3}, a_{4}\right) \mapsto\left(b_{1}, b_{2}, b_{3}, b_{4}\right)\right)\left(a_{2}\right)=b_{2}$, and

(iv) if $a_{1} \neq a_{2}$ and $a_{1} \neq a_{3}$ and $a_{1} \neq a_{4}$, then $\left(\left(a_{1}, a_{2}, a_{3}, a_{4}\right) \mapsto\right.$ $\left.\left(b_{1}, b_{2}, b_{3}, b_{4}\right)\right)\left(a_{1}\right)=b_{1}$.

(14) For all sets $a_{1}, a_{2}, a_{3}, b_{1}, b_{2}, b_{3}$ such that $a_{2} \neq a_{3}$ and $a_{1} \neq a_{2}$ and $a_{1} \neq a_{3}$ holds $\operatorname{rng}\left(\left(a_{1}, a_{2}, a_{3}\right) \mapsto\left(b_{1}, b_{2}, b_{3}\right)\right)=\left\{b_{1}, b_{2}, b_{3}\right\}$.

(15) For all sets $a_{1}, a_{2}, a_{3}, a_{4}, b_{1}, b_{2}, b_{3}, b_{4}$ such that $a_{2} \neq a_{3}$ and $a_{1} \neq a_{2}$ and $a_{1} \neq a_{3}$ and $a_{4} \neq a_{1}$ and $a_{4} \neq a_{2}$ and $a_{4} \neq a_{3}$ holds $\operatorname{rng}\left(\left(a_{1}, a_{2}, a_{3}, a_{4}\right) \mapsto\right.$ $\left.\left(b_{1}, b_{2}, b_{3}, b_{4}\right)\right)=\left\{b_{1}, b_{2}, b_{3}, b_{4}\right\}$.

(16) For every set $X$ and for all sets $a_{1}, a_{2}, a_{3}$ such that $a_{1}, a_{2}, a_{3} \in X$ holds $\left\{a_{1}, a_{2}, a_{3}\right\} \subseteq X$.

(17) For every set $X$ and for all sets $a_{1}, a_{2}, a_{3}, a_{4}$ such that $a_{1}, a_{2}, a_{3}, a_{4} \in X$ holds $\left\{a_{1}, a_{2}, a_{3}, a_{4}\right\} \subseteq X$.

(18) Let $X$ be a set and $a_{1}, a_{2}, a_{3}, a_{4}, a_{5}, a_{6}$ be sets. If $a_{1}, a_{2}, a_{3}, a_{4}, a_{5}$, $a_{6} \in X$, then $\left\{a_{1}, a_{2}, a_{3}, a_{4}, a_{5}, a_{6}\right\} \subseteq X$.

(19) Let $X$ be a set and $a_{1}, a_{2}, a_{3}, a_{4}, a_{5}, a_{6}, a_{7}, a_{8}, a_{9}$ be sets. Suppose $a_{1}, a_{2}$, $a_{3}, a_{4}, a_{5}, a_{6}, a_{7}, a_{8}, a_{9} \in X$. Then $\left\{a_{1}, a_{2}, a_{3}, a_{4}, a_{5}, a_{6}, a_{7}, a_{8}, a_{9}\right\} \subseteq X$.

(20) Let $X$ be a set and $a_{1}, a_{2}, a_{3}, a_{4}, a_{5}, a_{6}, a_{7}, a_{8}, a_{9}, a_{10}$ be sets. Suppose $a_{1}, a_{2}, a_{3}, a_{4}, a_{5}, a_{6}, a_{7}, a_{8}, a_{9}, a_{10} \in X$. Then $\left\{a_{1}, a_{2}, a_{3}, a_{4}, a_{5}, a_{6}, a_{7}, a_{8}, a_{9}, a_{10}\right\} \subseteq X$.

(21) For all sets $a_{1}, a_{2}, a_{3}, a_{4}, a_{5}, a_{6}, a_{7}, a_{8}, a_{9}$ holds $\left\{a_{1}\right\} \cup$ $\left\{a_{2}, a_{3}, a_{4}, a_{5}, a_{6}, a_{7}, a_{8}, a_{9}\right\}=\left\{a_{1}, a_{2}, a_{3}, a_{4}, a_{5}, a_{6}, a_{7}, a_{8}, a_{9}\right\}$.

(22) For all sets $a_{1}, a_{2}, a_{3}, a_{4}, a_{5}, a_{6}, a_{7}, a_{8}, a_{9}, a_{10}$ holds $\left\{a_{1}\right\} \cup$ $\left\{a_{2}, a_{3}, a_{4}, a_{5}, a_{6}, a_{7}, a_{8}, a_{9}, a_{10}\right\}=\left\{a_{1}, a_{2}, a_{3}, a_{4}, a_{5}, a_{6}, a_{7}, a_{8}, a_{9}, a_{10}\right\}$. 
(23) For all sets $a_{1}, a_{2}, a_{3}, a_{4}, a_{5}, a_{6}, a_{7}, a_{8}, a_{9}$ holds $\left\{a_{1}, a_{2}, a_{3}, a_{4}, a_{5}, a_{6}, a_{7}, a_{8}\right\} \cup\left\{a_{9}\right\}=\left\{a_{1}, a_{2}, a_{3}, a_{4}, a_{5}, a_{6}, a_{7}, a_{8}, a_{9}\right\}$.

(24) For all sets $a_{1}, a_{2}, a_{3}, a_{4}, a_{5}, a_{6}, a_{7}, a_{8}, a_{9}, a_{10}$ holds $\left\{a_{1}, a_{2}, a_{3}, a_{4}, a_{5}, a_{6}, a_{7}, a_{8}, a_{9}\right\} \cup\left\{a_{10}\right\}=$ $\left\{a_{1}, a_{2}, a_{3}, a_{4}, a_{5}, a_{6}, a_{7}, a_{8}, a_{9}, a_{10}\right\}$.

(25) For all sets $a_{1}, a_{2}, a_{3}, a_{4}, a_{5}, a_{6}, a_{7}, a_{8}, a_{9}$ holds $\left\{a_{1}, a_{2}, a_{3}\right\} \cup$ $\left\{a_{4}, a_{5}, a_{6}, a_{7}, a_{8}, a_{9}\right\}=\left\{a_{1}, a_{2}, a_{3}, a_{4}, a_{5}, a_{6}, a_{7}, a_{8}, a_{9}\right\}$.

(26) For all sets $a_{1}, a_{2}, a_{3}, a_{4}$ such that $a_{1} \neq a_{2}$ and $a_{1} \neq a_{3}$ and $a_{1} \neq a_{4}$ and $a_{2} \neq a_{3}$ and $a_{2} \neq a_{4}$ and $a_{3} \neq a_{4}$ holds $\left\langle a_{1}, a_{2}, a_{3}, a_{4}\right\rangle$ is one-to-one.

Let $a_{1}, a_{2}, a_{3}, a_{4}, a_{5}, a_{6}$ be sets. The functor $\left\langle a_{1}, a_{2}, a_{3}, a_{4}, a_{5}, a_{6}\right\rangle$ yielding a finite sequence is defined as follows:

(Def. 4) $\left\langle a_{1}, a_{2}, a_{3}, a_{4}, a_{5}, a_{6}\right\rangle=\left\langle a_{1}, a_{2}, a_{3}, a_{4}, a_{5}\right\rangle \frown\left\langle a_{6}\right\rangle$.

Let $X$ be a non empty set and let $a_{1}, a_{2}, a_{3}, a_{4}, a_{5}, a_{6}$ be elements of $X$. Then $\left\langle a_{1}, a_{2}, a_{3}, a_{4}, a_{5}, a_{6}\right\rangle$ is a finite sequence of elements of $X$.

Let $a_{1}, a_{2}, a_{3}, a_{4}, a_{5}, a_{6}$ be sets. One can check that $\left\langle a_{1}, a_{2}, a_{3}, a_{4}, a_{5}, a_{6}\right\rangle$ is 6-element.

We now state two propositions:

(27) Let $a_{1}, a_{2}, a_{3}, a_{4}, a_{5}, a_{6}$ be sets and $f$ be a finite sequence. Then $f=$ $\left\langle a_{1}, a_{2}, a_{3}, a_{4}, a_{5}, a_{6}\right\rangle$ if and only if the following conditions are satisfied:

len $f=6$ and $f(1)=a_{1}$ and $f(2)=a_{2}$ and $f(3)=a_{3}$ and $f(4)=a_{4}$ and $f(5)=a_{5}$ and $f(6)=a_{6}$.

(28) For all sets $a_{1}, a_{2}, a_{3}, a_{4}, a_{5}, a_{6}$ holds $\operatorname{rng}\left\langle a_{1}, a_{2}, a_{3}, a_{4}, a_{5}, a_{6}\right\rangle=$ $\left\{a_{1}, a_{2}, a_{3}, a_{4}, a_{5}, a_{6}\right\}$.

Let $a_{1}, a_{2}, a_{3}, a_{4}, a_{5}, a_{6}, a_{7}$ be sets. The functor $\left\langle a_{1}, a_{2}, a_{3}, a_{4}, a_{5}, a_{6}, a_{7}\right\rangle$ yields a finite sequence and is defined by:

(Def. 5) $\left\langle a_{1}, a_{2}, a_{3}, a_{4}, a_{5}, a_{6}, a_{7}\right\rangle=\left\langle a_{1}, a_{2}, a_{3}, a_{4}, a_{5}\right\rangle \frown\left\langle a_{6}, a_{7}\right\rangle$.

Let $X$ be a non empty set and let $a_{1}, a_{2}, a_{3}, a_{4}, a_{5}, a_{6}, a_{7}$ be elements of $X$. Then $\left\langle a_{1}, a_{2}, a_{3}, a_{4}, a_{5}, a_{6}, a_{7}\right\rangle$ is a finite sequence of elements of $X$.

Let $a_{1}, a_{2}, a_{3}, a_{4}, a_{5}, a_{6}, a_{7}$ be sets. Observe that $\left\langle a_{1}, a_{2}, a_{3}, a_{4}, a_{5}, a_{6}, a_{7}\right\rangle$ is 7-element.

We now state two propositions:

(29) Let $a_{1}, a_{2}, a_{3}, a_{4}, a_{5}, a_{6}, a_{7}$ be sets and $f$ be a finite sequence. Then $f=\left\langle a_{1}, a_{2}, a_{3}, a_{4}, a_{5}, a_{6}, a_{7}\right\rangle$ if and only if the following conditions are satisfied:

len $f=7$ and $f(1)=a_{1}$ and $f(2)=a_{2}$ and $f(3)=a_{3}$ and $f(4)=a_{4}$ and $f(5)=a_{5}$ and $f(6)=a_{6}$ and $f(7)=a_{7}$.

(30) For all sets $a_{1}, a_{2}, a_{3}, a_{4}, a_{5}, a_{6}, a_{7}$ holds $\operatorname{rng}\left\langle a_{1}, a_{2}, a_{3}, a_{4}, a_{5}, a_{6}, a_{7}\right\rangle=$ $\left\{a_{1}, a_{2}, a_{3}, a_{4}, a_{5}, a_{6}, a_{7}\right\}$.

Let $a_{1}, a_{2}, a_{3}, a_{4}, a_{5}, a_{6}, a_{7}, a_{8}$ be sets. The functor $\left\langle a_{1}, a_{2}, a_{3}, a_{4}, a_{5}, a_{6}, a_{7}, a_{8}\right\rangle$ yielding a finite sequence is defined by: 
(Def. 6) $\left\langle a_{1}, a_{2}, a_{3}, a_{4}, a_{5}, a_{6}, a_{7}, a_{8}\right\rangle=\left\langle a_{1}, a_{2}, a_{3}, a_{4}, a_{5}\right\rangle \frown\left\langle a_{6}, a_{7}, a_{8}\right\rangle$.

Let $X$ be a non empty set and let $a_{1}, a_{2}, a_{3}, a_{4}, a_{5}, a_{6}, a_{7}, a_{8}$ be elements of $X$. Then $\left\langle a_{1}, a_{2}, a_{3}, a_{4}, a_{5}, a_{6}, a_{7}, a_{8}\right\rangle$ is a finite sequence of elements of $X$.

Let $a_{1}, a_{2}, a_{3}, a_{4}, a_{5}, a_{6}, a_{7}, a_{8}$ be sets.

Observe that $\left\langle a_{1}, a_{2}, a_{3}, a_{4}, a_{5}, a_{6}, a_{7}, a_{8}\right\rangle$ is 8-element.

The following propositions are true:

(31) Let $a_{1}, a_{2}, a_{3}, a_{4}, a_{5}, a_{6}, a_{7}, a_{8}$ be sets and $f$ be a finite sequence. Then $f=\left\langle a_{1}, a_{2}, a_{3}, a_{4}, a_{5}, a_{6}, a_{7}, a_{8}\right\rangle$ if and only if the following conditions are satisfied:

len $f=8$ and $f(1)=a_{1}$ and $f(2)=a_{2}$ and $f(3)=a_{3}$ and $f(4)=a_{4}$ and $f(5)=a_{5}$ and $f(6)=a_{6}$ and $f(7)=a_{7}$ and $f(8)=a_{8}$.

(32) For all sets $a_{1}, a_{2}, a_{3}, a_{4}, a_{5}, a_{6}, a_{7}, a_{8}$ holds $\operatorname{rng}\left\langle a_{1}, a_{2}, a_{3}, a_{4}, a_{5}, a_{6}, a_{7}, a_{8}\right\rangle=\left\{a_{1}, a_{2}, a_{3}, a_{4}, a_{5}, a_{6}, a_{7}, a_{8}\right\}$.

(33) For all sets $a_{1}, a_{2}, a_{3}, a_{4}, a_{5}, a_{6}, a_{7}, a_{8}, a_{9}$ holds $\operatorname{rng}\left(\left\langle a_{1}, a_{2}, a_{3}, a_{4}, a_{5}, a_{6}, a_{7}, a_{8}\right\rangle^{\frown}\left\langle a_{9}\right\rangle\right)=\left\{a_{1}, a_{2}, a_{3}, a_{4}, a_{5}, a_{6}, a_{7}, a_{8}, a_{9}\right\}$.

(34) $\operatorname{Seg} 9=\{1,2,3,4,5,6,7,8,9\}$.

(35) $\operatorname{Seg} 10=\{1,2,3,4,5,6,7,8,9,10\}$.

We now state the proposition

(36) Let $a_{1}, a_{2}, a_{3}, a_{4}, a_{5}, a_{6}, a_{7}, a_{8}, a_{9}$ be sets. Then dom $w_{9}=\operatorname{Seg} 9$ and $w_{9}(1)=a_{1}$ and $w_{9}(2)=a_{2}$ and $w_{9}(3)=a_{3}$ and $w_{9}(4)=a_{4}$ and $w_{9}(5)=a_{5}$ and $w_{9}(6)=a_{6}$ and $w_{9}(7)=a_{7}$ and $w_{9}(8)=a_{8}$ and $w_{9}(9)=a_{9}$, where $w_{9}=\left\langle a_{1}, a_{2}, a_{3}, a_{4}, a_{5}, a_{6}, a_{7}, a_{8}\right\rangle^{\frown}\left\langle a_{9}\right\rangle$.

The following proposition is true

(37) Let $a_{1}, a_{2}, a_{3}, a_{4}, a_{5}, a_{6}, a_{7}, a_{8}, a_{9}, a_{10}$ be sets. Then dom $w_{10}=\operatorname{Seg} 10$ and $w_{10}(1)=a_{1}$ and $w_{10}(2)=a_{2}$ and $w_{10}(3)=a_{3}$ and $w_{10}(4)=a_{4}$ and $w_{10}(5)=a_{5}$ and $w_{10}(6)=a_{6}$ and $w_{10}(7)=a_{7}$ and $w_{10}(8)=a_{8}$ and $w_{10}(9)=a_{9}$ and $w_{10}(10)=a_{10}$, where $w_{10}=\left\langle a_{1}, a_{2}, a_{3}, a_{4}, a_{5}, a_{6}, a_{7}, a_{8}\right\rangle^{\frown}$ $\left\langle a_{9}, a_{10}\right\rangle$.

Let $I, J$ be sets and let $\Sigma$ be a many sorted set indexed by $I$. A many sorted function indexed by $I$ is said to be a double many sorted set of $\Sigma$ and $J$ if:

(Def. 7) For all sets $i, j$ such that $i \in I$ holds $\operatorname{domit}(i)=\Sigma(i)$ and if $j \in \Sigma(i)$, then $\operatorname{it}(i)(j)$ is a many sorted set indexed by $J$.

Let $I, J$ be sets, let $\Sigma_{1}$ be a many sorted set indexed by $I$, and let $\Sigma_{2}$ be a many sorted set indexed by $J$. A double many sorted set of $\Sigma_{1}$ and $J$ is said to be a double many sorted set of $\Sigma_{1}$ and $\Sigma_{2}$ if:

(Def. 8) For all sets $i, a$ such that $i \in I$ and $a \in \Sigma_{1}(i)$ holds $\operatorname{it}(i)(a)$ is a many sorted subset of $\Sigma_{2}$.

Let $I$ be a set, let $X, Y$ be many sorted sets indexed by $I$, let $f$ be a double many sorted set of $X$ and $Y$, and let $x, y$ be sets. Note that $f(x)(y)$ is function-like and relation-like. 
Let $\Sigma$ be a many sorted signature, let $o, a$ be sets, and let $r$ be an element of $\Sigma$. We say that $o$ is of type $a \rightarrow r$ if and only if:

(Def. 9) (The arity of $\Sigma)(o)=a$ and (the result sort of $\Sigma)(o)=r$.

One can prove the following propositions:

(38) Let $\Sigma$ be a non void non empty many sorted signature, $o$ be an operation symbol of $\Sigma$, and $r$ be a sort symbol of $\Sigma$. Suppose $o$ is of type $\emptyset \rightarrow r$. Let $\mathfrak{A}$ be an algebra over $\Sigma$. Suppose (the sorts of $\mathfrak{A})(r) \neq \emptyset$. Then (Den $(o(\in$ the carrier' of $\Sigma), \mathfrak{A}))(\emptyset)$ is an element of (the sorts of $\mathfrak{A})(r)$.

(39) Let $\Sigma$ be a non void non empty many sorted signature, $o, a$ be sets, and $r$ be a sort symbol of $\Sigma$. Suppose $o$ is of type $\langle a\rangle \rightarrow r$. Let $\mathfrak{A}$ be an algebra over $\Sigma$. Suppose (the sorts of $\mathfrak{A})(a) \neq \emptyset$ and (the sorts of $\mathfrak{A})(r) \neq \emptyset$. Let $x$ be an element of (the sorts of $\mathfrak{A})(a)$. Then (Den $(o(\in$ the carrier' of $\Sigma)$, $\mathfrak{A}))(\langle x\rangle)$ is an element of (the sorts of $\mathfrak{A})(r)$.

(40) Let $\Sigma$ be a non void non empty many sorted signature, $o, a, b$ be sets, and $r$ be a sort symbol of $\Sigma$. Suppose $o$ is of type $\langle a, b\rangle \rightarrow r$. Let $\mathfrak{A}$ be an algebra over $\Sigma$. Suppose (the sorts of $\mathfrak{A})(a) \neq \emptyset$ and (the sorts of $\mathfrak{A})(b) \neq \emptyset$ and (the sorts of $\mathfrak{A})(r) \neq \emptyset$. Let $x$ be an element of (the sorts of $\mathfrak{A})(a)$ and $y$ be an element of (the sorts of $\mathfrak{A})(b)$. Then (Den $(o(\in$ the carrier' of $\Sigma)$, $\mathfrak{A}))(\langle x, y\rangle)$ is an element of (the sorts of $\mathfrak{A})(r)$.

(41) Let $\Sigma$ be a non void non empty many sorted signature, $o, a, b, c$ be sets, and $r$ be a sort symbol of $\Sigma$. Suppose $o$ is of type $\langle a, b, c\rangle \rightarrow r$. Let $\mathfrak{A}$ be an algebra over $\Sigma$. Suppose (the sorts of $\mathfrak{A})(a) \neq \emptyset$ and (the sorts of $\mathfrak{A})(b) \neq \emptyset$ and (the sorts of $\mathfrak{A})(c) \neq \emptyset$ and (the sorts of $\mathfrak{A})(r) \neq \emptyset$. Let $x$ be an element of (the sorts of $\mathfrak{A})(a), y$ be an element of (the sorts of $\mathfrak{A})(b)$, and $z$ be an element of (the sorts of $\mathfrak{A})(c)$. Then (Den $(o(\in$ the carrier' of $\Sigma), \mathfrak{A}))(\langle x, y, z\rangle)$ is an element of (the sorts of $\mathfrak{A})(r)$.

(42) Let $\Sigma_{1}, \Sigma_{2}$ be many sorted signatures. Suppose the many sorted signature of $\Sigma_{1}=$ the many sorted signature of $\Sigma_{2}$. Let $o, a$ be sets, $r_{1}$ be an element of $\Sigma_{1}$, and $r_{2}$ be an element of $\Sigma_{2}$. If $r_{1}=r_{2}$, then if $o$ is of type $a \rightarrow r_{1}$, then $o$ is of type $a \rightarrow r_{2}$.

(43) Let $o$ be an operation symbol of $\Sigma, r$ be a sort symbol of $\Sigma$, and $\mathfrak{A}$ be an algebra over $\Sigma$. If $o$ is of type $\emptyset \rightarrow r$, then $\emptyset \in \operatorname{Args}(o, \mathfrak{A})$.

(44) Let $o$ be an operation symbol of $\Sigma, s, r$ be sort symbols of $\Sigma$, and $\mathfrak{A}$ be an algebra over $\Sigma$. If $o$ is of type $\langle s\rangle \rightarrow r$ and $x \in($ the sorts of $\mathfrak{A})(s)$, then $\langle x\rangle \in \operatorname{Args}(o, \mathfrak{A})$.

(45) Let $o$ be an operation symbol of $\Sigma, s_{1}, s_{2}, r$ be sort symbols of $\Sigma$, and $\mathfrak{A}$ be an algebra over $\Sigma$. Suppose $o$ is of type $\left\langle s_{1}, s_{2}\right\rangle \rightarrow r$ and $x \in$ (the sorts of $\mathfrak{A})\left(s_{1}\right)$ and $y \in($ the sorts of $\mathfrak{A})\left(s_{2}\right)$. Then $\langle x, y\rangle \in \operatorname{Args}(o, \mathfrak{A})$.

(46) Let $o$ be an operation symbol of $\Sigma, s_{1}, s_{2}, s_{3}, r$ be sort symbols of $\Sigma$, and $\mathfrak{A}$ be an algebra over $\Sigma$. Suppose $o$ is of type $\left\langle s_{1}, s_{2}, s_{3}\right\rangle \rightarrow r$ and $x \in$ (the 
sorts of $\mathfrak{A})\left(s_{1}\right)$ and $y \in($ the sorts of $\mathfrak{A})\left(s_{2}\right)$ and $z \in($ the sorts of $\mathfrak{A})\left(s_{3}\right)$. Then $\langle x, y, z\rangle \in \operatorname{Args}(o, \mathfrak{A})$.

\section{Free Variables}

Let $\Sigma$ be a non empty non void many sorted signature. We consider free variable algebras over $\Sigma$ as extensions of algebra over $\Sigma$ as systems

$\langle$ sorts, a characteristics, free variables 〉,

where the sorts constitute a many sorted set indexed by the carrier of $\Sigma$, the characteristics is a many sorted function from the sorts $\#$. the arity of $\Sigma$ into the sorts the result sort of $\Sigma$, and the free variables constitute a double many sorted set of the sorts and the sorts.

Let $\Sigma$ be a non empty non void many sorted signature, let $U$ be a non-empty many sorted set indexed by the carrier of $\Sigma$, let $C$ be a many sorted function from $U^{\#}$. the arity of $\Sigma$ into $U$. the result sort of $\Sigma$, and let $v$ be a double many sorted set of $U$ and $U$. Observe that $\langle U, C, v\rangle_{V}$ is non-empty.

Let $\Sigma$ be a non empty non void many sorted signature and let $X$ be a nonempty many sorted set indexed by the carrier of $\Sigma$. Observe that there exists a strict free variable algebra over $\Sigma$ which is non-empty and including $\Sigma$-terms over $X$.

Let $\Sigma$ be a non empty non void many sorted signature. One can check that there exists a free variable algebra over $\Sigma$ which is non-empty and disjoint valued. Let $X$ be a non-empty many sorted set indexed by the carrier of $\Sigma$. One can check that every including $\Sigma$-terms over $X$ free variable algebra over $\Sigma$ which has all variables is also non-empty.

Let $\Sigma$ be a non empty non void many sorted signature, let $\mathfrak{A}$ be a non-empty free variable algebra over $\Sigma$, let $a$ be a sort symbol of $\Sigma$, and let $t$ be an element of $\mathfrak{A}$ from $a$. The functor vf $t$ yields a many sorted subset of the sorts of $\mathfrak{A}$ and is defined as follows:

(Def. 10) $\quad$ vf $t=($ the free variables of $\mathfrak{A})(a)(t)$.

Let $\Sigma$ be a non empty non void many sorted signature and let $\mathfrak{A}$ be a nonempty free variable algebra over $\Sigma$. We say that $\mathfrak{A}$ is vf-correct if and only if the condition (Def. 11) is satisfied.

(Def. 11) Let $o$ be an operation symbol of $\Sigma$ and $p$ be a finite sequence. Suppose $p \in \operatorname{Args}(o, \mathfrak{A})$. Let $b$ be an element of $\mathfrak{A}$ from the result sort of $o$. Suppose $b=(\operatorname{Den}(o, \mathfrak{A}))(p)$. Let $s$ be a sort symbol of $\Sigma$. Then $(\operatorname{vf} b)(s) \subseteq \bigcup\left\{(\operatorname{vf} a)(s) ; s_{0}\right.$ ranges over sort symbols of $\Sigma$, $a$ ranges over elements of $\mathfrak{A}$ from $s_{0}: \bigvee_{i \text { :natural number }}\left(i \in \operatorname{dom} \operatorname{Arity}(o) \wedge s_{0}=\right.$ $\operatorname{Arity}(o)(i) \wedge a=p(i))\}$.

Next we state three propositions: 
(47) Let $\Sigma$ be a non empty non void many sorted signature and $\mathfrak{A}, \mathfrak{B}$ be algebras over $\Sigma$. Suppose the algebra of $\mathfrak{A}=$ the algebra of $\mathfrak{B}$. Let $G$ be a subset of $\mathfrak{A}$ and $H$ be a subset of $\mathfrak{B}$. If $G=H$, then $\operatorname{Gen}(G)=\operatorname{Gen}(H)$.

(48) Let $\Sigma$ be a non empty non void many sorted signature and $\mathfrak{A}, \mathfrak{B}$ be algebras over $\Sigma$. Suppose the algebra of $\mathfrak{A}=$ the algebra of $\mathfrak{B}$. Then every generator set of $\mathfrak{A}$ is a generator set of $\mathfrak{B}$.

(49) Let $\Sigma$ be a non empty non void many sorted signature and $\mathfrak{A}, \mathfrak{B}$ be non-empty algebras over $\Sigma$. Suppose the algebra of $\mathfrak{A}=$ the algebra of $\mathfrak{B}$. Let $G$ be a generator set of $\mathfrak{A}$ and $H$ be a generator set of $\mathfrak{B}$. If $G=H$, then if $G$ is free, then $H$ is free.

Let $\Sigma$ be a non empty non void many sorted signature and let $X$ be a nonempty many sorted set indexed by the carrier of $\Sigma$. Observe that there exists a non-empty including $\Sigma$-terms over $X$ strict free variable algebra over $\Sigma$ which is free in itself, has all variables, and inherits operations.

Let $\Sigma$ be a non empty non void many sorted signature, let $X$ be a non-empty many sorted set indexed by the carrier of $\Sigma$, and let $\mathfrak{A}$ be a non-empty including $\Sigma$-terms over $X$ free variable algebra over $\Sigma$. We say that $\mathfrak{A}$ is vf-free if and only if the condition (Def. 12) is satisfied.

(Def. 12) Let $s, r$ be sort symbols of $\Sigma$ and $t$ be an element of $\mathfrak{A}$ from $s$. Then $(\operatorname{vf} t)(r)=\left\{t\left\lceil p ; p\right.\right.$ ranges over elements of $\operatorname{dom} t:\left(t\lceil p)(\emptyset)_{\mathbf{2}}=r\right\}$.

The scheme Scheme deals with a non empty set $\mathcal{A}$, non-empty many sorted sets $\mathcal{B}, \mathcal{C}$ indexed by $\mathcal{A}$, and a ternary functor $\mathcal{F}$ yielding a set, and states that: There exists a double many sorted set $f$ of $\mathcal{B}$ and $\mathcal{C}$ such that for all elements $s, r$ of $\mathcal{A}$ and for every element $t$ of $\mathcal{B}(s)$ holds $f(s)(t)(r)=\mathcal{F}(s, r, t)$

provided the parameters satisfy the following condition:

- For all elements $s, r$ of $\mathcal{A}$ and for every element $t$ of $\mathcal{B}(s)$ holds $\mathcal{F}(s, r, t)$ is a subset of $\mathcal{C}(r)$.

Next we state the proposition

(50) Let $\Sigma$ be a non empty non void many sorted signature, $X$ be a nonempty many sorted set indexed by the carrier of $\Sigma$, and $\mathfrak{A}$ be a free in itself including $\Sigma$-terms over $X$ algebra over $\Sigma$ with all variables and inheriting operations. Then there exists a double many sorted set $V_{1}$ of the sorts of $\mathfrak{A}$ and the sorts of $\mathfrak{A}$ and there exists a free in itself including $\Sigma$-terms over $X$ free variable algebra $\mathfrak{B}$ over $\Sigma$ with all variables and inheriting operations such that $\mathfrak{B}=\left\langle\text { the sorts of } \mathfrak{A} \text {, the characteristics of } \mathfrak{A}, V_{1}\right\rangle_{V}$ and $\mathfrak{B}$ is vf-free.

Let $\Sigma$ be a non empty non void many sorted signature and let $X$ be a nonempty many sorted set indexed by the carrier of $\Sigma$. One can verify that there exists a free in itself including $\Sigma$-terms over $X$ free variable algebra over $\Sigma$ with all variables and inheriting operations which is strict and vf-free. 
We now state two propositions:

(51) Let $\Sigma$ be a non empty non void many sorted signature, $X$ be a non-empty many sorted set indexed by the carrier of $\Sigma, \mathfrak{A}$ be a vf-free including $\Sigma$ terms over $X$ free variable algebra over $\Sigma$ with all variables and inheriting operations, $s$ be a sort symbol of $\Sigma$, and $t$ be an element of $\mathfrak{A}$ from $s$. Then vf $t$ is a many sorted subset of FreeGenerator $(X)$.

(52) Let $\Sigma$ be a non empty non void many sorted signature, $X$ be a non-empty many sorted set indexed by the carrier of $\Sigma, \mathfrak{A}$ be a vf-free non-empty including $\Sigma$-terms over $X$ free variable algebra over $\Sigma, s$ be a sort symbol of $\Sigma$, and $x$ be an element of $\mathfrak{A}$ from $s$. If $x \in($ FreeGenerator $(X))(s)$, then vf $x=s$-singleton $x$.

\section{Algebra with Undefined Values}

Let $I$ be a set and let $\Sigma$ be a many sorted set indexed by $I$. A many sorted element of $\Sigma$ is an element of $\Sigma$.

Let $I$ be a non empty set, let $A$ be a non-empty many sorted set indexed by $I$, let $e$ be a many sorted element of $A$, and let $i$ be an element of $I$. Then $e(i)$ is an element of $A(i)$.

Let $\Sigma$ be a non empty non void many sorted signature. We introduce algebras over $\Sigma$ with undefined values which are extensions of algebra over $\Sigma$ and are systems

〈sorts, a characteristics, an undefined map 〉, where the sorts constitute a many sorted set indexed by the carrier of $\Sigma$, the characteristics is a many sorted function from the sorts $\#$. the arity of $\Sigma$ into the sorts the result sort of $\Sigma$, and the undefined map is a many sorted element of the sorts.

Let $\Sigma$ be a non empty non void many sorted signature. Note that there exists an algebra over $\Sigma$ with undefined values which is non-empty.

Let $\Sigma$ be a non empty non void many sorted signature, let $\mathfrak{A}$ be an algebra over $\Sigma$ with undefined values, let $s$ be a sort symbol of $\Sigma$, and let $a$ be an element of $\mathfrak{A}$ from $s$. We say that $a$ is undefined if and only if:

(Def. 13) $\quad a=($ the undefined map of $\mathfrak{A})(s)$.

Let $\Sigma$ be a non empty non void many sorted signature, let $\mathfrak{A}$ be an algebra over $\Sigma$, let $s$ be a sort symbol of $\Sigma$, and let $a$ be an element of $\mathfrak{A}$ from $s$. We say that $a$ is defined if and only if:

(Def. 14) For every algebra $\mathfrak{B}$ over $\Sigma$ with undefined values such that $\mathfrak{B}=\mathfrak{A}$ holds $a \neq($ the undefined map of $\mathfrak{B})(s)$.

Let $\Sigma$ be a non empty non void many sorted signature and let $\mathfrak{A}$ be an algebra over $\Sigma$. The defined sorts of $\mathfrak{A}$ constitute a many sorted subset of the sorts of $\mathfrak{A}$ defined by: 
(Def. 15)(i) For every algebra $\mathfrak{B}$ over $\Sigma$ with undefined values such that $\mathfrak{A}=\mathfrak{B}$ and for every many sorted set $U$ indexed by the carrier of $\Sigma$ such that for every sort symbol $s$ of $\Sigma$ holds $U(s)=\{($ the undefined map of $\mathfrak{B})(s)\}$ holds the defined sorts of $\mathfrak{A}=($ the sorts of $\mathfrak{A}) \backslash U$ if $\mathfrak{A}$ is an algebra over $\Sigma$ with undefined values,

(ii) the defined sorts of $\mathfrak{A}=$ the sorts of $\mathfrak{A}$, otherwise.

We now state the proposition

(53) Let $\Sigma_{1}, \Sigma_{2}$ be non empty non void many sorted signatures, $\mathfrak{A}_{1}$ be an algebra over $\Sigma_{1}$ with undefined values, and $\mathfrak{A}_{2}$ be an algebra over $\Sigma_{2}$ with undefined values. Suppose the sorts of $\mathfrak{A}_{1}=$ the sorts of $\mathfrak{A}_{2}$ and the undefined map of $\mathfrak{A}_{1}=$ the undefined map of $\mathfrak{A}_{2}$. Then the defined sorts of $\mathfrak{A}_{1}=$ the defined sorts of $\mathfrak{A}_{2}$.

Let $\Sigma$ be a non empty non void many sorted signature and let $\mathfrak{A}$ be an algebra over $\Sigma$. We say that $\mathfrak{A}$ has defined elements if and only if:

(Def. 16) The defined sorts of $\mathfrak{A}$ are non-empty.

Let $\Sigma$ be a non empty non void many sorted signature, let $\mathfrak{A}$ be a non-empty algebra over $\Sigma$ with undefined values, let $s$ be a sort symbol of $\Sigma$, and let $a$ be an element of $\mathfrak{A}$ from $s$. Let us observe that $a$ is defined if and only if:

(Def. 17) $\quad a \in($ the defined sorts of $\mathfrak{A})(s)$.

Let $\Sigma$ be a non empty non void many sorted signature and let $\mathfrak{A}$ be an algebra over $\Sigma$ with undefined values. We say that $\mathfrak{A}$ is undefined consequently if and only if the condition (Def. 18) is satisfied.

(Def. 18) Let $o$ be an operation symbol of $\Sigma$ and $p$ be a finite sequence. Suppose that

(i) $\quad p \in \operatorname{Args}(o, \mathfrak{A})$, and

(ii) there exists a natural number $i$ and there exists a sort symbol $s$ of $\Sigma$ and there exists an element $a$ of $\mathfrak{A}$ from $s$ such that $i \in \operatorname{dom} \operatorname{Arity}(o)$ and $s=\operatorname{Arity}(o)(i)$ and $a=p(i)$ and $a$ is undefined.

Let $b$ be an element of $\mathfrak{A}$ from the result sort of $o$. If $b=(\operatorname{Den}(o, \mathfrak{A}))(p)$, then $b$ is undefined.

Let $I$ be a set and let $A$ be a many sorted set indexed by $I$. The functor succ $A$ yielding a many sorted set indexed by $I$ is defined as follows:

(Def. 19) For every set $i$ such that $i \in I$ holds $(\operatorname{succ} A)(i)=\operatorname{succ} A(i)$.

Let $I$ be a set and let $A$ be a many sorted set indexed by $I$. Note that succ $A$ is non-empty.

Let $\Sigma$ be a non empty non void many sorted signature, let $\mathfrak{A}$ be an algebra over $\Sigma$, and let $\mathfrak{B}$ be an algebra over $\Sigma$ with undefined values. We say that $\mathfrak{B}$ is $\mathfrak{A}$ with undefined values if and only if the conditions (Def. 20) are satisfied.

(Def. 20)(i) $\quad \mathfrak{B}$ is undefined consequently,

(ii) the undefined map of $\mathfrak{B}=$ the sorts of $\mathfrak{A}$, 
(iii) the sorts of $\mathfrak{B}=\operatorname{succ}($ the sorts of $\mathfrak{A}$ ), and

(iv) for every operation symbol $o$ of $\Sigma$ and for every element $a$ of $\operatorname{Args}(o, \mathfrak{A})$ such that $\operatorname{Args}(o, \mathfrak{A}) \neq \emptyset$ holds if $(\operatorname{Den}(o, \mathfrak{B}))(a) \neq(\operatorname{Den}(o, \mathfrak{A}))(a)$, then $(\operatorname{Den}(o, \mathfrak{B}))(a)=($ the undefined map of $\mathfrak{B})($ the result sort of $o)$.

We now state the proposition

(54) Let $\Sigma$ be a non empty non void many sorted signature, $\mathfrak{A}$ be an algebra over $\Sigma$, and $\mathfrak{B}$ be an algebra over $\Sigma$ with undefined values. Suppose $\mathfrak{B}$ is $\mathfrak{A}$ with undefined values. Then the defined sorts of $\mathfrak{B}=$ the sorts of $\mathfrak{A}$.

Let $\Sigma$ be a non empty many sorted signature and let $\mathfrak{A}$ be an algebra over $\Sigma$. Observe that the characteristics of $\mathfrak{A}$ is function yielding.

Let $\Sigma$ be a non empty non void many sorted signature. Note that every algebra over $\Sigma$ which has defined elements is also non-empty.

The scheme UndefAlgebra deals with a non empty non void many sorted signature $\mathcal{A}$, a non-empty algebra $\mathcal{B}$ over $\mathcal{A}$, and a binary predicate $\mathcal{P}$, and states that:

There exists a strict algebra $\mathfrak{B}$ over $\mathcal{A}$ with undefined values such that

(i) $\mathfrak{B}$ is $\mathcal{B}$ with undefined values and has defined elements,

(ii) the undefined map of $\mathfrak{B}=$ the sorts of $\mathcal{B}$,

(iii) the sorts of $\mathfrak{B}=\operatorname{succ}($ the sorts of $\mathcal{B}$ ), and

(iv) for every operation symbol $o$ of $\mathcal{A}$ and for every element $a$ of $\operatorname{Args}(o, \mathcal{B})$ holds if not $\mathcal{P}[o, a]$, then $(\operatorname{Den}(o, \mathfrak{B}))(a)=(\operatorname{Den}(o$, $\mathcal{B}))(a)$ and if $\mathcal{P}[o, a]$, then $(\operatorname{Den}(o, \mathfrak{B}))(a)=($ the undefined map of $\mathfrak{B}$ )(the result sort of $o$ )

for all values of the parameters.

One can prove the following proposition

(55) Let $\mathfrak{A}$ be a non-empty algebra over $\Sigma$. Then there exists a strict algebra $\mathfrak{B}$ over $\Sigma$ with undefined values such that

(i) $\mathfrak{B}$ is $\mathfrak{A}$ with undefined values and has defined elements,

(ii) the undefined map of $\mathfrak{B}=$ the sorts of $\mathfrak{A}$,

(iii) the sorts of $\mathfrak{B}=\operatorname{succ}($ the sorts of $\mathfrak{A}$ ), and

(iv) for every operation symbol $o$ of $\Sigma$ and for every element $a$ of $\operatorname{Args}(o, \mathfrak{A})$ holds $(\operatorname{Den}(o, \mathfrak{B}))(a)=(\operatorname{Den}(o, \mathfrak{A}))(a)$.

Let $\Sigma$ be a non empty non void many sorted signature and let $\mathfrak{A}$ be a nonempty algebra over $\Sigma$. Note that every algebra over $\Sigma$ with undefined values which is $\mathfrak{A}$ with undefined values is also undefined consequently and there exists a strict algebra over $\Sigma$ with undefined values which is $\mathfrak{A}$ with undefined values and has defined elements.

Let $\Sigma$ be a non empty non void many sorted signature. One can verify that there exists an algebra over $\Sigma$ which has defined elements. 
Let $\Sigma$ be a non empty non void many sorted signature and let $\mathfrak{A}$ be an algebra over $\Sigma$ with defined elements. One can verify that the defined sorts of $\mathfrak{A}$ is non-empty. Let $s$ be a sort symbol of $\Sigma$. Note that there exists an element of $\mathfrak{A}$ from $s$ which is defined.

Let us consider $\Sigma$, let $\mathfrak{A}$ be an algebra over $\Sigma$ with undefined values with defined elements, and let $s$ be a sort symbol of $\Sigma$. Note that there exists an element of $\mathfrak{A}$ from $s$ which is defined.

\section{Program Algebra}

Let $J$ be a non empty non void many sorted signature, let $\mathfrak{T}$ be an algebra over $J$, and let $X$ be a generator set of $\mathfrak{T}$. We introduce program algebra structures of $J, \mathfrak{T}$, and $X$ which are extensions of universal algebra structures and are systems

$\langle$ a carrier, a characteristic, assignments $\rangle$, where the carrier is a set, the characteristic is a finite sequence of operational functions of the carrier, and the assignments constitute a function from $\bigcup \llbracket X$, the sorts of $\mathfrak{T} \rrbracket$ into the carrier.

Let $J$ be a non empty non void many sorted signature, let $\mathfrak{T}$ be an algebra over $J$, let $X$ be a generator set of $\mathfrak{T}$, and let $A$ be a program algebra structure of $J, \mathfrak{T}$, and $X$. We say that $A$ is disjoint valued if and only if:

(Def. 21) The sorts of $\mathfrak{T}$ are disjoint valued and the assignments of $A$ are one-toone.

Let $J$ be a non empty non void many sorted signature, let $\mathfrak{T}$ be an algebra over $J$, and let $X$ be a generator set of $\mathfrak{T}$. Note that there exists a strict program algebra structure of $J, \mathfrak{T}$, and $X$ which is partial, quasi total, and non-empty.

Let $J$ be a non empty non void many sorted signature, let $\mathfrak{T}$ be an algebra over $J$, and let $X$ be a generator set of $\mathfrak{T}$. Note that there exists a partial quasi total non-empty non empty strict program algebra structure of $J, \mathfrak{T}$, and $X$ which has empty-instruction, catenation, if-instruction, and while-instruction.

We now state several propositions:

(56) Let $U_{1}, U_{2}$ be pre-if-while algebras. Suppose the universal algebra structure of $U_{1}=$ the universal algebra structure of $U_{2}$. Then

(i) $\operatorname{EmptyIns}_{\left(U_{1}\right)}=\operatorname{EmptyIns}_{\left(U_{2}\right)}$, and

(ii) for all elements $I_{1}, J_{1}$ of $U_{1}$ and for all elements $I_{2}, J_{2}$ of $U_{2}$ such that $I_{1}=I_{2}$ and $J_{1}=J_{2}$ holds $I_{1} ; J_{1}=I_{2} ; J_{2}$ and while $I_{1}$ do $J_{1}=$ while $I_{2}$ do $J_{2}$ and for every element $C_{1}$ of $U_{1}$ and for every element $C_{2}$ of $U_{2}$ such that $C_{1}=C_{2}$ holds if $C_{1}$ then $I_{1}$ else $J_{1}=$ if $C_{2}$ then $I_{2}$ else $J_{2}$.

(57) Let $U_{1}, U_{2}$ be pre-if-while algebras. Suppose the universal algebra structure of $U_{1}=$ the universal algebra structure of $U_{2}$. Then ElementaryInstructions $_{\left(U_{1}\right)}=$ ElementaryInstructions $_{\left(U_{2}\right)}$. 
(58) Let $U_{1}, U_{2}$ be universal algebras, $\Sigma_{1}$ be a subset of $U_{1}$, and $\Sigma_{2}$ be a subset of $U_{2}$. Suppose $\Sigma_{1}=\Sigma_{2}$. Let $o_{1}$ be an operation of $U_{1}$ and $o_{2}$ be an operation of $U_{2}$. If $o_{1}=o_{2}$, then if $\Sigma_{1}$ is closed on $o_{1}$, then $\Sigma_{2}$ is closed on $o_{2}$.

(59) Let $U_{1}, U_{2}$ be universal algebras. Suppose the universal algebra structure of $U_{1}=$ the universal algebra structure of $U_{2}$. Let $\Sigma_{1}$ be a subset of $U_{1}$ and $\Sigma_{2}$ be a subset of $U_{2}$. If $\Sigma_{1}=\Sigma_{2}$, then if $\Sigma_{1}$ is operations closed, then $\Sigma_{2}$ is operations closed.

(60) Let $U_{1}, U_{2}$ be universal algebras. Suppose the universal algebra structure of $U_{1}=$ the universal algebra structure of $U_{2}$. Then every generator set of $U_{1}$ is a generator set of $U_{2}$.

(61) Let $U_{1}, U_{2}$ be universal algebras. Suppose the universal algebra structure of $U_{1}=$ the universal algebra structure of $U_{2}$. Then signature $U_{1}=$ signature $U_{2}$.

Let $J$ be a non empty non void many sorted signature, let $\mathfrak{T}$ be an algebra over $J$, and let $X$ be a generator set of $\mathfrak{T}$. Note that there exists a partial quasi total non-empty non empty strict program algebra structure of $J, \mathfrak{T}$, and $X$ with empty-instruction, catenation, if-instruction, and while-instruction which is non degenerated, well founded, E.C.I.W.-strict, and infinite.

Let $J$ be a non empty non void many sorted signature, let $\mathfrak{T}$ be an algebra over $J$, and let $X$ be a generator set of $\mathfrak{T}$. A pre-if-while algebra over $X$ is a partial quasi total non-empty non empty program algebra structure of $J, \mathfrak{T}$, and $X$ with empty-instruction, catenation, if-instruction, and while-instruction.

Let $J$ be a non empty non void many sorted signature, let $\mathfrak{T}$ be an algebra over $J$, and let $X$ be a generator set of $\mathfrak{T}$. A if-while algebra over $X$ is a non degenerated well founded E.C.I.W.-strict pre-if-while algebra over $X$.

Let $J$ be a non empty non void many sorted signature, let $\mathfrak{T}$ be a non-empty algebra over $J$, let $X$ be a non-empty generator set of $\mathfrak{T}$, let $A$ be a non empty program algebra structure of $J, \mathfrak{T}$, and $X$, let $a$ be a sort symbol of $J$, let $x$ be an element of $X(a)$, and let $t$ be an element of $\mathfrak{T}$ from $a$. The functor $x:={ }_{A} t$ yielding an algorithm of $A$ is defined as follows:

(Def. 22) $\quad x:={ }_{A} t=($ the assignments of $A)(\langle x, t\rangle)$.

Let $\Sigma$ be a set and let $\mathfrak{T}$ be a disjoint valued non-empty many sorted set indexed by $\Sigma$. Note that there exists a many sorted subset of $\mathfrak{T}$ which is nonempty.

Let $J$ be a non void non empty many sorted signature, let $\mathfrak{T}, \mathfrak{C}$ be non-empty algebras over $J$, and let $X$ be a non-empty generator set of $\mathfrak{T}$. The functor $\mathfrak{C}$-States $(X)$ yields a subset of $\operatorname{MSFuncs}(X$, the sorts of $\mathfrak{C})$ and is defined by the condition (Def. 23).

(Def. 23) Let $s$ be a many sorted function from $X$ into the sorts of $\mathfrak{C}$. Then $s \in$ $\mathfrak{C}$-States $(X)$ if and only if there exists a many sorted function $f$ from $\mathfrak{T}$ 
into $\mathfrak{C}$ such that $f$ is a homomorphism of $\mathfrak{T}$ into $\mathfrak{C}$ and $s=f\lceil X$.

Let $J$ be a non void non empty many sorted signature, let $\mathfrak{T}$ be a non-empty algebra over $J$, let $\mathfrak{C}$ be a non-empty image of $\mathfrak{T}$, and let $X$ be a non-empty generator set of $\mathfrak{T}$. One can verify that $\mathfrak{C}$-States $(X)$ is non empty.

The following proposition is true

(62) Let $B$ be a non void non empty many sorted signature, $\mathfrak{T}, \mathfrak{C}$ be nonempty algebras over $B, X$ be a non-empty generator set of $\mathfrak{T}$, and $g$ be a set. Suppose $g \in \mathfrak{C}$-States $(X)$. Then $g$ is a many sorted function from $X$ into the sorts of $\mathfrak{C}$.

Let $B$ be a non void non empty many sorted signature, let $\mathfrak{T}, \mathfrak{C}$ be nonempty algebras over $B$, and let $X$ be a non-empty generator set of $\mathfrak{T}$. Note that every element of $\mathfrak{C}$-States $(X)$ is relation-like and function-like.

Let $B$ be a non void non empty many sorted signature, let $\mathfrak{T}, \mathfrak{C}$ be nonempty algebras over $B$, and let $X$ be a non-empty generator set of $\mathfrak{T}$. One can check that every element of $\mathfrak{C}$-States $(X)$ is function yielding and the carrier of $B$-defined.

Let $B$ be a non void non empty many sorted signature, let $\mathfrak{T}$ be a non-empty algebra over $B$, let $\mathfrak{C}$ be a non-empty image of $\mathfrak{T}$, and let $X$ be a non-empty generator set of $\mathfrak{T}$. Observe that every element of $\mathfrak{C}$-States $(X)$ is total.

Let $B$ be a non void non empty many sorted signature, let $\mathfrak{T}$ be a non-empty algebra over $B$, let $\mathfrak{C}$ be a non-empty algebra over $B$, let $X$ be a non-empty generator set of $\mathfrak{T}$, let $a$ be a sort symbol of $B$, let $x$ be an element of $X(a)$, and let $f$ be an element of $\mathfrak{C}$ from $a$. The functor $\operatorname{States}_{x \rightarrow f}(X)$ yields a subset of $\mathfrak{C}$-States $(X)$ and is defined by the condition (Def. 24).

(Def. 24) Let $s$ be a many sorted function from $X$ into the sorts of $\mathfrak{C}$. Then $s \in$ States $_{x \rightarrow f}(X)$ if and only if $s \in \mathfrak{C}-\operatorname{States}(X)$ and $s(a)(x) \neq f$.

Let $\Sigma$ be a non empty non void many sorted signature, let $\mathfrak{A}$ be a nonempty algebra over $\Sigma$, and let $o$ be an operation symbol of $\Sigma$. Observe that every element of $\operatorname{Args}(o, \mathfrak{A})$ is function-like and relation-like.

Let $B$ be a non void non empty many sorted signature, let $X$ be a non-empty many sorted set indexed by the carrier of $B$, let $\mathfrak{T}$ be an including $B$-terms over $X$ non-empty algebra over $B$, let $\mathfrak{C}$ be a non-empty image of $\mathfrak{T}$, let $a$ be a sort symbol of $B$, let $t$ be an element of $\mathfrak{T}$ from $a$, and let $s$ be a function yielding function. Let us assume that there exist a many sorted function $h$ from $\mathfrak{T}$ into $\mathfrak{C}$ and a generator set $Q$ of $\mathfrak{T}$ such that $h$ is a homomorphism of $\mathfrak{T}$ into $\mathfrak{C}$ and $Q=\operatorname{dom}_{\kappa} s(\kappa)$ and $s=h \uparrow Q$. The functor $t$ value at $(\mathfrak{C}, s)$ yielding an element of $\mathfrak{C}$ from $a$ is defined by the condition (Def. 25).

(Def. 25) There exists a many sorted function $f$ from $\mathfrak{T}$ into $\mathfrak{C}$ and there exists a generator set $Q$ of $\mathfrak{T}$ such that $f$ is a homomorphism of $\mathfrak{T}$ into $\mathfrak{C}$ and $Q=\operatorname{dom}_{\kappa} s(\kappa)$ and $s=f \uparrow Q$ and $t$ value at $(\mathfrak{C}, s)=f(a)(t)$. 


\section{Generator System}

Let us consider $\Sigma, X$ and let $\mathfrak{T}$ be a non-empty including $\Sigma$-terms over $X$ algebra over $\Sigma$. We introduce generator systems over $\Sigma, X$, and $\mathfrak{T}$ which are systems

〈generators, a supported variable, a supported term 〉, where the generators constitute a non-empty generator set of $\mathfrak{T}$, the supported variable is a many sorted function from the generators into FreeGenerator $(X)$, and the supported term is a double many sorted set of the generators and the carrier of $\Sigma$.

Let us consider $\Sigma, X$, let $\mathfrak{T}$ be a non-empty including $\Sigma$-terms over $X$ algebra over $\Sigma$, let $G$ be a generator system over $\Sigma, X$, and $\mathfrak{T}$, and let $s$ be a sort symbol of $\Sigma$. An element of $\mathfrak{T}$ from $s$ is said to be an element of $G$ from $s$ if:

(Def. 26) $\quad$ It $\in($ the generators of $G)(s)$.

Let us consider $\Sigma, X$, let $\mathfrak{T}$ be a non-empty including $\Sigma$-terms over $X$ algebra over $\Sigma$, let $G$ be a generator system over $\Sigma, X$, and $\mathfrak{T}$, and let $s$ be a sort symbol of $\Sigma$. The functor $G(s)$ yields a component of the generators of $G$ and is defined by:

(Def. 27) $G(s)=($ the generators of $G)(s)$.

Let $g$ be an element of $G$ from $s$. The functor supp-var $g$ yielding an element of $($ FreeGenerator $(X))(s)$ is defined as follows:

(Def. 28) supp-var $g=($ the supported variable of $G)(s)(g)$.

Let us consider $\Sigma, X$, let $\mathfrak{T}$ be a non-empty including $\Sigma$-terms over $X$ free variable algebra over $\Sigma$, let $G$ be a generator system over $\Sigma, X$, and $\mathfrak{T}$, let $s$ be a sort symbol of $\Sigma$, and let $g$ be an element of $G$ from $s$. Let us assume that (the supported term of $G)(s)(g)$ is a many sorted function from vf $g$ into the sorts of $\mathfrak{T}$. The functor supp-term $g$ yielding a many sorted function from vf $g$ into the sorts of $\mathfrak{T}$ is defined as follows:

(Def. 29) supp-term $g=($ the supported term of $G)(s)(g)$.

Let $\Sigma$ be a non void non empty many sorted signature, let $X$ be a non-empty many sorted set indexed by the carrier of $\Sigma$, let $\mathfrak{T}$ be a non-empty including $\Sigma$-terms over $X$ free variable algebra over $\Sigma$, let $\mathfrak{C}$ be a non-empty image of $\mathfrak{T}$, and let $G$ be a generator system over $\Sigma, X$, and $\mathfrak{T}$. We say that $G$ is $\mathfrak{C}$-supported if and only if the conditions (Def. 30) are satisfied.

(Def. 30)(i) FreeGenerator $(X)$ is a many sorted subset of the generators of $G$, and

(ii) for every sort symbol $s$ of $\Sigma$ holds dom(the supported term of $G)(s)=G(s)$ and for every element $t$ of $G$ from $s$ holds (the supported term of $G)(s)(t)$ is a many sorted function from vf $t$ into the sorts of $\mathfrak{T}$ and if $t \in($ FreeGenerator $(X))(s)$, then supp-term $t=\mathrm{id}_{s \text {-singleton } t}$ and supp-var $t=t$ and for every element $v$ of $\mathfrak{C}$-States(the generators of $G$ ) such that $v(s)(\operatorname{supp}-\operatorname{var} t)=v(s)(t)$ and for every sort symbol $r$ of $\Sigma$ and 
for every element $x$ of (FreeGenerator $(X))(r)$ and for every element $q$ of (the sorts of $\mathfrak{T})(r)$ such that $x \in(\operatorname{vf} t)(r)$ and $q=(\operatorname{supp}$-term $t)(r)(x)$ and $q$ value at $(\mathfrak{C}, v)$ is defined holds $v(r)(x)=q$ value at $(\mathfrak{C}, v)$ and if $t \notin($ FreeGenerator $(X))(s)$, then for every many sorted subset $H$ of the generators of $G$ such that $H=$ FreeGenerator $(X)$ and for every element $v$ of $\mathfrak{C}$ from $s$ and for every many sorted function $f$ from the generators of $G$ into the sorts of $\mathfrak{C}$ such that $f \in \mathfrak{C}$-States(the generators of $G$ ) and for every many sorted function $u$ from FreeGenerator $(X)$ into the sorts of $\mathfrak{C}$ such that for every sort symbol $a$ of $\Sigma$ and for every element $z$ of $($ FreeGenerator $(X))(a)$ such that $z \in(\operatorname{vf} t)(a)$ and for every element $q$ of $\mathfrak{T}$ from $a$ such that $q=(\operatorname{supp}$-term $t)(a)(z)$ holds $u(a)(z)=q$ value at $(\mathfrak{C},(f \uparrow H)+\cdot(s$, supp-var $t, v))$ and for every many sorted subset $H$ of the sorts of $\mathfrak{T}$ such that $H=$ FreeGenerator $(X)$ and for every many sorted function $h$ from $\mathfrak{T}$ into $\mathfrak{C}$ such that $h$ is a homomorphism of $\mathfrak{T}$ into $\mathfrak{C}$ and $h \uparrow H=u$ holds $v=h(s)(t)$.

Let us consider $\Sigma$, let us consider $X$, let $\mathfrak{A}$ be a vf-free free in itself including $\Sigma$-terms over $X$ free variable algebra over $\Sigma$ with all variables and inheriting operations, let $\mathfrak{C}$ be a non-empty image of $\mathfrak{A}$, and let $G$ be a generator system over $\Sigma, X$, and $\mathfrak{A}$. Let us assume that $G$ is $\mathfrak{C}$-supported. Let $s$ be an element of $\mathfrak{C}$-States (the generators of $G$ ), let $r$ be a sort symbol of $\Sigma$, let $v$ be an element of $\mathfrak{C}$ from $r$, and let $t$ be an element of $G$ from $r$. The functor $\operatorname{succ}_{t:=v}(s)$ yields an element of $\mathfrak{C}$-States(the generators of $G$ ) and is defined by the conditions (Def. 31).

(Def. 31)(i) $\quad\left(\operatorname{succ}_{t:=v}(s)\right)(r)(t)=v$, and

(ii) for every sort symbol $p$ of $\Sigma$ and for every element $x$ of $($ FreeGenerator $(X))(p)$ such that if $p=r$, then $x \neq t$ holds if $x \notin(\operatorname{vf} t)(p)$, then $\left(\operatorname{succ}_{t:=v}(s)\right)(p)(x)=s(p)(x)$ and for every many sorted function $u$ from FreeGenerator $(X)$ into the sorts of $\mathfrak{C}$ and for every many sorted subset $H$ of the generators of $G$ such that $H=$ FreeGenerator $(X)$ and for every many sorted function $f$ from the generators of $G$ into the sorts of $\mathfrak{C}$ such that $f=s$ and $u=(f\lceil H)+\cdot(r$, supp-var $t, v)$ holds if $x \in(\operatorname{vf} t)(p)$, then for every element $q$ of $\mathfrak{A}$ from $p$ such that $q=(\operatorname{supp}$-term $t)(p)(x)$ holds $\left.\operatorname{succ}_{t:=v}(s)\right)(p)(x)=q$ value at $(\mathfrak{C}, u)$.

Let $B$ be a non void non empty many sorted signature, let $Y$ be a nonempty many sorted set indexed by the carrier of $B$, let $\mathfrak{T}$ be a vf-free free in itself including $B$-terms over $Y$ free variable algebra over $B$ with all variables and inheriting operations, let $\mathfrak{C}$ be a non-empty image of $\mathfrak{T}$, let $X$ be a generator system over $B, Y$, and $\mathfrak{T}$, let $A$ be a pre-if-while algebra over the generators of $X$, let $a$ be a sort symbol of $B$, let $x$ be an element of (the generators of $X)(a)$, and let $z$ be an element of $\mathfrak{C}$ from $a$. The functor $\mathfrak{C}$-Execution Ex $_{x \neq z}(A)$ yields a subset of $(\mathfrak{C}$-States (the generators of $\left.\left.X))^{(\mathfrak{C}-S t a t e s(t h e ~ g e n e r a t o r s ~ o f ~} X\right)\right) \times$ the carrier of $A$ and 
is defined by the condition (Def. 32).

(Def. 32) Let $f$ be a function from $(\mathfrak{C}$-States(the generators of $X)) \times$ the carrier of $A$ into $\mathfrak{C}$-States(the generators of $X)$. Then $f \in \mathfrak{C}$-Execution $\operatorname{Ex}_{x \rightarrow z}(A)$ if and only if $f$ is an execution function of $A$ over $\mathfrak{C}$-States(the generators of $X$ ) and States $_{x \rightarrow z}$ (the generators of $X$ ).

\section{Boolean Signature}

We consider connectives signatures as extensions of many sorted signature as systems

〈 a carrier, a carrier', an arity, a result sort, connectives 〉, where the carrier and the carrier' are sets, the arity is a function from the carrier' into the carrier*, the result sort is a function from the carrier' into the carrier, and the connectives constitute a finite sequence of elements of the carrier'.

Let $\Sigma$ be a connectives signature. We say that $\Sigma$ is 1 -1-connectives if and only if:

(Def. 33) The connectives of $\Sigma$ are one-to-one.

Let $n$ be a natural number and let $\Sigma$ be a connectives signature. We say that $\Sigma$ is $n$-connectives if and only if:

(Def. 34) len (the connectives of $\Sigma)=n$.

Let $n$ be a natural number. Note that there exists a strict connectives signature which is $n$-connectives, non empty, and non void.

We consider boolean signatures as extensions of connectives signature as systems

〈 a carrier, a carrier', an arity, a result sort, a boolean sort, connectives 〉, where the carrier and the carrier' are sets, the arity is a function from the carrier' into the carrier*, the result sort is a function from the carrier' into the carrier, the boolean sort is an element of the carrier, and the connectives constitute a finite sequence of elements of the carrier'.

Let $n$ be a natural number. Note that there exists a strict boolean signature which is $n$-connectives, non empty, and non void.

Let $B$ be a boolean signature. We say that $B$ is boolean correct if and only if the conditions (Def. 35) are satisfied.

(Def. 35)(i) len (the connectives of $B) \geq 3$,

(ii) (the connectives of $B)(1)$ is of type $\emptyset \rightarrow$ the boolean sort of $B$,

(iii) (the connectives of $B)(2)$ is of type $\langle$ the boolean sort of $B\rangle \rightarrow$ the boolean sort of $B$, and

(iv) (the connectives of $B)(3)$ is of type $\langle$ the boolean sort of $B$, the boolean sort of $B\rangle \rightarrow$ the boolean sort of $B$. 
One can verify that there exists a strict boolean signature which is 3connectives, 1-1-connectives, boolean correct, non empty, and non void.

Let us note that there exists a connectives signature which is 1-1-connectives, non empty, and non void.

Let $\Sigma$ be a 1-1-connectives non empty non void connectives signature. Note that the connectives of $\Sigma$ is one-to-one.

Let $\Sigma$ be a non empty non void boolean signature and let $\mathfrak{B}$ be an algebra over $\Sigma$. We say that $\mathfrak{B}$ is boolean correct if and only if the conditions (Def. 36) are satisfied.

(Def. 36)(i) (The defined sorts of $\mathfrak{B})($ the boolean sort of $\Sigma)=$ Boolean,

(ii) $\quad($ Den $(($ the connectives of $\Sigma)(1)(\in$ the carrier' of $\Sigma), \mathfrak{B}))(\emptyset)=$ true, and

(iii) for all boolean sets $x, y$ holds (Den $(($ the connectives of $\Sigma)(2)(\in$ the carrier' of $\Sigma), \mathfrak{B}))(\langle x\rangle)=\neg x$ and $(\operatorname{Den}(($ the connectives of $\Sigma)(3)(\in$ the carrier' of $\Sigma), \mathfrak{B}))(\langle x, y\rangle)=x \wedge y$.

One can prove the following proposition

(63) Let $A, B$ be non empty sets, $n$ be a natural number, and $f$ be a function from $A^{n}$ into $B$. Then

(i) $f$ is a homogeneous quasi total non empty partial function from $A^{*}$ to $B$, and

(ii) for every homogeneous function $g$ such that $f=g$ holds $g$ is $n$-ary.

Let $A, B$ be non empty sets and let $n$ be a natural number. Note that there exists a homogeneous quasi total non empty partial function from $A^{*}$ to $B$ which is $n$-ary.

Now we present two schemes. The scheme $S$ ch 1 deals with non empty sets $\mathcal{A}, \mathcal{B}$ and a unary functor $\mathcal{F}$ yielding an element of $\mathcal{B}$, and states that:

There exists a 1-ary homogeneous quasi total non empty partial function $f$ from $\mathcal{A}^{*}$ to $\mathcal{B}$ such that for every element $a$ of $\mathcal{A}$ holds $f(\langle a\rangle)=\mathcal{F}(a)$

for all values of the parameters.

The scheme $S$ ch2 deals with non empty sets $\mathcal{A}, \mathcal{B}$ and a binary functor $\mathcal{F}$ yielding an element of $\mathcal{B}$, and states that:

There exists a 2-ary homogeneous quasi total non empty partial function $f$ from $\mathcal{A}^{*}$ to $\mathcal{B}$ such that for all elements $a, b$ of $\mathcal{A}$ holds $f(\langle a, b\rangle)=\mathcal{F}(a, b)$

for all values of the parameters.

One can prove the following propositions:

(64) Let $\Sigma$ be a non empty non void many sorted signature, $A$ be a non-empty many sorted set indexed by the carrier of $\Sigma, f$ be a many sorted function from $A^{\#}$. the arity of $\Sigma$ into $A$. the result sort of $\Sigma, o$ be an operation symbol of $\Sigma$, and $d$ be a function from $\left(A^{\#}\right.$. the arity of $\left.\Sigma\right)(o)$ into $(A \cdot$ the 
result sort of $\Sigma)(o)$. Then $f+\cdot(o, d)$ is a many sorted function from $A^{\#}$. the arity of $\Sigma$ into $A \cdot$ the result sort of $\Sigma$.

(65) Let $\Sigma$ be a boolean correct non empty non void boolean signature and $A$ be a non-empty many sorted set indexed by the carrier of $\Sigma$. Then there exists a strict algebra $\mathfrak{B}$ over $\Sigma$ with undefined values with defined elements such that

(i) the defined sorts of $\mathfrak{B}=A+\cdot$ (the boolean sort of $\Sigma$, Boolean),

(ii) the undefined map of $\mathfrak{B}=$ the defined sorts of $\mathfrak{B}$,

(iii) the sorts of $\mathfrak{B}=\operatorname{succ}($ the defined sorts of $\mathfrak{B}$ ), and

(iv) $\mathfrak{B}$ is boolean correct and undefined consequently.

Let $\Sigma$ be a boolean correct non empty non void boolean signature. One can verify that there exists a strict algebra over $\Sigma$ with undefined values which is boolean correct and undefined consequently and has defined elements and there exists an algebra over $\Sigma$ which is boolean correct and has defined elements.

Let $\Sigma$ be a boolean correct non empty non void boolean signature and let $\mathfrak{B}$ be a non-empty algebra over $\Sigma$. The functor true $\mathfrak{B}$ yielding an element of $\mathfrak{B}$ from the boolean sort of $\Sigma$ is defined as follows:

(Def. 37) $\operatorname{true}_{\mathfrak{B}}=(\operatorname{Den}(($ the connectives of $\Sigma)(1)(\in$ the carrier' of $\Sigma), \mathfrak{B}))(\emptyset)$.

Let $p$ be an element of $\mathfrak{B}$ from the boolean sort of $\Sigma$. The functor $\neg p$ yields an element of $\mathfrak{B}$ from the boolean sort of $\Sigma$ and is defined as follows:

(Def. 38) $\neg p=(\operatorname{Den}(($ the connectives of $\Sigma)(2)(\in$ the carrier' of $\Sigma), \mathfrak{B}))(\langle p\rangle)$.

Let $q$ be an element of $\mathfrak{B}$ from the boolean sort of $\Sigma$. The functor $p \wedge q$ yielding an element of $\mathfrak{B}$ from the boolean sort of $\Sigma$ is defined as follows:

(Def. 39) $p \wedge q=(\operatorname{Den}(($ the connectives of $\Sigma)(3)(\in$ the carrier' of $\Sigma), \mathfrak{B}))(\langle p, q\rangle)$.

Let $\Sigma$ be a boolean correct non empty non void boolean signature and let $\mathfrak{B}$ be a non-empty algebra over $\Sigma$. The functor false $\mathfrak{B}$ yielding an element of $\mathfrak{B}$ from the boolean sort of $\Sigma$ is defined as follows:

(Def. 40) false $_{\mathfrak{B}}=\neg$ true $_{\mathfrak{B}}$.

Let $p$ be an element of $\mathfrak{B}$ from the boolean sort of $\Sigma$ and let $q$ be an element of $\mathfrak{B}$ from the boolean sort of $\Sigma$. The functor $p \vee q$ yields an element of $\mathfrak{B}$ from the boolean sort of $\Sigma$ and is defined by:

(Def. 41) $\quad p \vee q=\neg(\neg p \wedge \neg q)$.

The functor $p \Rightarrow q$ yielding an element of $\mathfrak{B}$ from the boolean sort of $\Sigma$ is defined by:

(Def. 42) $p \Rightarrow q=\neg(p \wedge \neg q)$.

Let $\Sigma$ be a boolean correct non empty non void boolean signature, let $\mathfrak{B}$ be a non-empty algebra over $\Sigma$, let $p$ be an element of $\mathfrak{B}$ from the boolean sort of $\Sigma$, and let $q$ be an element of $\mathfrak{B}$ from the boolean sort of $\Sigma$. The functor $p \Leftrightarrow q$ yielding an element of $\mathfrak{B}$ from the boolean sort of $\Sigma$ is defined by:

(Def. 43) $\quad p \Leftrightarrow q=(p \wedge q) \vee(\neg p \wedge \neg q)$. 
The following proposition is true

(66) Let $\Sigma$ be a boolean correct non empty non void boolean signature and $\mathfrak{B}$ be a boolean correct algebra over $\Sigma$ with undefined values with defined elements. Then

(i) true $_{\mathfrak{B}}=$ true,

(ii) false $_{\mathfrak{B}}=$ false, and

(iii) for all defined elements $x, y$ of $\mathfrak{B}$ from the boolean sort of $\Sigma$ and for all boolean numbers $a, b$ such that $a=x$ and $b=y$ holds $\neg x=\neg a$ and $x \wedge y=a \wedge b$ and $x \vee y=a \vee b$ and $x \Rightarrow y=a \Rightarrow b$ and $x \Leftrightarrow y=a \Leftrightarrow b$.

\section{Algebra with Integers}

Let $i$ be a natural number, let $s$ be a set, and let $\Sigma$ be a boolean signature. We say that $\Sigma$ has integers with connectives from $i$ and the sort at $s$ if and only if the conditions (Def. 44) are satisfied.

(Def. 44)(i) len (the connectives of $\Sigma) \geq i+6$, and

(ii) there exists an element $I$ of $\Sigma$ such that $I=s$ and $I \neq$ the boolean sort of $\Sigma$ and (the connectives of $\Sigma$ ) $(i)$ is of type $\emptyset \rightarrow I$ and (the connectives of $\Sigma)(i+1)$ is of type $\emptyset \rightarrow I$ and (the connectives of $\Sigma)(i) \neq($ the connectives of $\Sigma)(i+1)$ and (the connectives of $\Sigma)(i+2)$ is of type $\langle I\rangle \rightarrow I$ and (the connectives of $\Sigma)(i+3)$ is of type $\langle I, I\rangle \rightarrow I$ and (the connectives of $\Sigma)(i+4)$ is of type $\langle I, I\rangle \rightarrow I$ and (the connectives of $\Sigma)(i+5)$ is of type $\langle I, I\rangle \rightarrow I$ and (the connectives of $\Sigma)(i+3) \neq$ (the connectives of $\Sigma)(i+4)$ and (the connectives of $\Sigma)(i+3) \neq($ the connectives of $\Sigma)(i+5)$ and (the connectives of $\Sigma)(i+4) \neq($ the connectives of $\Sigma)(i+5)$ and (the connectives of $\Sigma)(i+6)$ is of type $\langle I, I\rangle \rightarrow$ the boolean sort of $\Sigma$.

The following proposition is true

(67) There exists an 10-connectives non empty non void strict boolean signature $\Sigma$ such that

(i) $\Sigma$ is 1-1-connectives and boolean correct and has integers with connectives from 4 and the sort at 1 ,

(ii) the carrier of $\Sigma=\{0,1\}$, and

(iii) there exists a sort symbol $I$ of $\Sigma$ such that $I=1$ and (the connectives of $\Sigma)(4)$ is of type $\emptyset \rightarrow I$.

Let us mention that there exists a strict boolean signature which is 10connectives, 1-1-connectives, boolean correct, non empty, and non void and has integers with connectives from 4 and the sort at 1 .

Let $\Sigma$ be a non empty non void boolean signature, let $N$ be a set, and let $I$ be a sort symbol of $\Sigma$. We say that $I$ is integer sort of $N$ if and only if:

(Def. 45) $\quad I=N$. 
Let $\Sigma$ be a non empty non void boolean signature and let $I$ be a sort symbol of $\Sigma$. We say that $I$ is integer if and only if:

(Def. 46) $I$ is integer sort of 1.

Let $\Sigma$ be a non empty non void boolean signature. Observe that every sort symbol of $\Sigma$ which is integer is also integer sort of 1 and every sort symbol of $\Sigma$ which is integer sort of 1 is also integer.

Let $\Sigma$ be a non empty non void boolean signature with integers with connectives from 4 and the sort at 1 . One can verify that there exists a sort symbol of $\Sigma$ which is integer.

We now state the proposition

(68) Let $\Sigma$ be a non empty non void boolean signature with integers with connectives from 4 and the sort at 1 and $I$ be an integer sort symbol of $\Sigma$. Then $I \neq$ the boolean sort of $\Sigma$ and (the connectives of $\Sigma)(4)$ is of type $\emptyset \rightarrow I$ and (the connectives of $\Sigma)(4+1)$ is of type $\emptyset \rightarrow I$ and (the connectives of $\Sigma)(4) \neq($ the connectives of $\Sigma)(4+1)$ and (the connectives of $\Sigma)(4+2)$ is of type $\langle I\rangle \rightarrow I$ and (the connectives of $\Sigma)(4+3)$ is of type $\langle I, I\rangle \rightarrow I$ and (the connectives of $\Sigma)(4+4)$ is of type $\langle I, I\rangle \rightarrow I$ and (the connectives of $\Sigma)(4+5)$ is of type $\langle I, I\rangle \rightarrow I$ and (the connectives of $\Sigma)(4+3) \neq($ the connectives of $\Sigma)(4+4)$ and (the connectives of $\Sigma)(4+3) \neq$ (the connectives of $\Sigma)(4+5)$ and (the connectives of $\Sigma)(4+4) \neq($ the connectives of $\Sigma)(4+5)$ and (the connectives of $\Sigma)(4+6)$ is of type $\langle I, I\rangle$ $\rightarrow$ the boolean sort of $\Sigma$.

Let $\Sigma$ be a non empty non void boolean signature with integers with connectives from 4 and the sort at 1 , let $\mathfrak{A}$ be a non-empty algebra over $\Sigma$, and let $I$ be an integer sort symbol of $\Sigma$. The functor $0_{\mathfrak{A}}^{I}$ yields an element of (the sorts of $\mathfrak{A})(I)$ and is defined by:

(Def. 47) $\quad 0_{\mathfrak{A}}^{I}=(\operatorname{Den}(($ the connectives of $\Sigma)(4)(\in$ the carrier' of $\Sigma), \mathfrak{A}))(\emptyset)$.

The functor $1_{\mathfrak{A}}^{I}$ yields an element of (the sorts of $\left.\mathfrak{A}\right)(I)$ and is defined as follows:

(Def. 48) $\quad 1_{\mathfrak{A}}^{I}=(\operatorname{Den}(($ the connectives of $\Sigma)(5)(\in$ the carrier' of $\Sigma), \mathfrak{A}))(\emptyset)$.

Let $a$ be an element of (the sorts of $\mathfrak{A})(I)$. The functor $-a$ yielding an element of (the sorts of $\mathfrak{A})(I)$ is defined as follows:

(Def. 49) $-a=(\operatorname{Den}(($ the connectives of $\Sigma)(6)(\in$ the carrier' of $\Sigma), \mathfrak{A}))(\langle a\rangle)$.

Let $b$ be an element of (the sorts of $\mathfrak{A})(I)$. The functor $a+b$ yielding an element of (the sorts of $\mathfrak{A})(I)$ is defined as follows:

(Def. 50$) \quad a+b=(\operatorname{Den}(($ the connectives of $\Sigma)(7)(\in$ the carrier' of $\Sigma), \mathfrak{A}))(\langle a, b\rangle)$.

The functor $a \cdot b$ yielding an element of (the sorts of $\mathfrak{A})(I)$ is defined as follows:

(Def. 51) $\quad a \cdot b=(\operatorname{Den}(($ the connectives of $\Sigma)(8)(\in$ the carrier' of $\Sigma), \mathfrak{A}))(\langle a, b\rangle)$.

The functor $a \operatorname{div} b$ yielding an element of (the sorts of $\mathfrak{A})(I)$ is defined by:

(Def. 52) $\quad a \operatorname{div} b=(\operatorname{Den}(($ the connectives of $\Sigma)(9)(\in$ the carrier' of $\Sigma), \mathfrak{A}))(\langle a, b\rangle)$. 
The functor leq $(a, b)$ yielding an element of (the sorts of $\mathfrak{A}$ )(the boolean sort of $\Sigma)$ is defined by:

(Def. 53) $\operatorname{leq}(a, b)=(\operatorname{Den}(($ the connectives of $\Sigma)(10)(\in$ the carrier' of $\Sigma), \mathfrak{A}))(\langle a$, $b\rangle)$.

Let $\Sigma$ be a non empty non void boolean signature with integers with connectives from 4 and the sort at 1 , let $\mathfrak{A}$ be a non-empty algebra over $\Sigma$, let $I$ be an integer sort symbol of $\Sigma$, and let $a, b$ be elements of $\mathfrak{A}$ from $I$. The functor $a-b$ yields an element of $\mathfrak{A}$ from $I$ and is defined by:

(Def. 54) $a-b=a+-b$.

The functor $a$ mod $b$ yields an element of $\mathfrak{A}$ from $I$ and is defined by:

(Def. 55) $a \bmod b=a+-(a \operatorname{div} b) \cdot b$.

Let $\Sigma$ be a non empty non void boolean signature with integers with connectives from 4 and the sort at 1 and let $X$ be a non-empty many sorted set indexed by the carrier of $\Sigma$. One can verify that $X(1)$ is non empty.

Let $n$ be a natural number, let $s$ be a set, let $\Sigma$ be a boolean correct non empty non void boolean signature, and let $\mathfrak{A}$ be a boolean correct algebra over $\Sigma$. We say that $\mathfrak{A}$ has integers with connectives from $n$ and the sort at $s$ if and only if the condition (Def. 56) is satisfied.

(Def. 56) There exists a sort symbol $I$ of $\Sigma$ such that

(i) $\quad I=s$,

(ii) (the connectives of $\Sigma)(n)$ is of type $\emptyset \rightarrow I$,

(iii) (the defined sorts of $\mathfrak{A})(I)=\mathbb{Z}$,

(iv) $\quad(\operatorname{Den}(($ the connectives of $\Sigma)(n)(\in$ the carrier' of $\Sigma), \mathfrak{A}))(\emptyset)=0$,

(v) $\quad(\operatorname{Den}(($ the connectives of $\Sigma)(n+1)(\in$ the carrier' of $\Sigma), \mathfrak{A}))(\emptyset)=1$, and

(vi) for all integers $i, j$ holds (Den $(($ the connectives of $\Sigma)(n+2)(\in$ the carrier' of $\Sigma), \mathfrak{A}))(\langle i\rangle)=-i$ and $(\operatorname{Den}(($ the connectives of $\Sigma)(n+3)(\in$ the carrier' of $\Sigma), \mathfrak{A}))(\langle i, j\rangle)=i+j$ and $(\operatorname{Den}(($ the connectives of $\Sigma)(n+4)(\in$ the carrier' of $\Sigma), \mathfrak{A}))(\langle i, j\rangle)=i \cdot j$ and if $j \neq 0$, then $(\operatorname{Den}(($ the connectives of $\Sigma)(n+5)(\in$ the carrier' of $\Sigma), \mathfrak{A}))(\langle i, j\rangle)=i \operatorname{div} j$ and (Den $(($ the connectives of $\Sigma)(n+6)(\in$ the carrier' of $\Sigma), \mathfrak{A}))(\langle i, j\rangle)=(i>j \rightarrow$ false, true $)$.

Let $\Sigma$ be a non empty non void boolean signature, let $I$ be a set, let $n$ be a natural number, and let $\mathfrak{A}$ be an algebra over $\Sigma$ with undefined values with defined elements. We say that $\mathfrak{A}$ has division by 0 undefined with $n$ and $I$ if and only if the condition (Def. 57) is satisfied.

(Def. 57) Let $J$ be a sort symbol of $\Sigma$. Suppose $I=J$. Let $a$ be a defined element of (the sorts of $\mathfrak{A})(J)$. Then (Den $(($ the connectives of $\Sigma)(n+5)(\in$ the carrier' of $\Sigma), \mathfrak{A}))(\langle a,(\operatorname{Den}(($ the connectives of $\Sigma)(n)(\in$ the carrier' of $\Sigma)$, $\mathfrak{A})(\emptyset)\rangle)=($ the undefined map of $\mathfrak{A})(J)$.

Let $\Sigma$ be a non empty non void boolean signature with integers with connectives from 4 and the sort at 1 and let $\mathfrak{A}$ be an algebra over $\Sigma$ with undefined 
values with defined elements. We say that $\mathfrak{A}$ has division by 0 undefined if and only if:

(Def. 58) $\mathfrak{A}$ has division by 0 undefined with 4 and 1.

Let $\Sigma$ be a non empty non void boolean signature with integers with connectives from 4 and the sort at 1 and let $\mathfrak{A}$ be an algebra over $\Sigma$ with undefined values with defined elements. Let us observe that $\mathfrak{A}$ has division by 0 undefined if and only if the condition (Def. 59) is satisfied.

(Def. 59) Let $I$ be an integer sort symbol of $\Sigma$ and $a$ be a defined element of (the sorts of $\mathfrak{A})(I)$. Then $a \operatorname{div} 0_{\mathfrak{A}}^{I}$ is undefined.

The following proposition is true

(69) Let $n$ be a natural number and $I$ be a set. Suppose $n \geq 1$. Let $\Sigma$ be a boolean correct non empty non void boolean signature. Suppose $\Sigma$ has integers with connectives from $n$ and the sort at $I$. Then there exists a boolean correct strict algebra $\mathfrak{A}$ over $\Sigma$ with undefined values with defined elements such that

(i) the undefined map of $\mathfrak{A}=$ the defined sorts of $\mathfrak{A}$,

(ii) the sorts of $\mathfrak{A}=\operatorname{succ}($ the defined sorts of $\mathfrak{A}$ ), and

(iii) $\mathfrak{A}$ is undefined consequently and has integers with connectives from $n$ and the sort at $I$ and division by 0 undefined with $n$ and $I$.

Let $\Sigma$ be a boolean correct non empty non void boolean signature with integers with connectives from 4 and the sort at 1 . Note that there exists a boolean correct strict algebra over $\Sigma$ with undefined values with defined elements which is undefined consequently and has integers with connectives from 4 and the sort at 1 and division by 0 undefined.

One can prove the following proposition

(70) Let $\Sigma$ be a boolean correct non empty non void boolean signature with integers with connectives from 4 and the sort at $1, \mathfrak{A}$ be a boolean correct algebra over $\Sigma$ with undefined values with integers with connectives from 4 and the sort at 1 and defined elements, and $I$ be an integer sort symbol of $\Sigma$. Then

(i) (the defined sorts of $\mathfrak{A})(I)=\mathbb{Z}$,

(ii) $0_{\mathfrak{A}}^{I}=0$,

(iii) $1_{\mathfrak{A}}^{I}=1$, and

(iv) for all integers $i, j$ and for all elements $a, b$ of (the sorts of $\mathfrak{A})(I)$ such that $a=i$ and $b=j$ holds $-a=-i$ and $a+b=i+j$ and $a-b=i-j$ and $a \cdot b=i \cdot j$ and if $j \neq 0$, then $a \operatorname{div} b=i \operatorname{div} j$ and $a \bmod b=i \bmod j$ and $\operatorname{leq}(a, b)=(i>j \rightarrow$ false, true $)$ and leq $(a, b)=$ true iff $i \leq j$ and $\operatorname{leq}(a, b)=$ false iff $i>j$. 


\section{Algebras with Arrays}

Let $I, N$ be sets, let $n$ be a natural number, and let $\Sigma$ be a connectives signature. We say that $\Sigma$ has arrays of type $I$ with connectives from $n$ and integers at $N$ if and only if the conditions (Def. 60) are satisfied.

(Def. 60)(i) len (the connectives of $\Sigma$ ) $\geq n+3$, and

(ii) there exist elements $J, K, L$ of $\Sigma$ such that $L=I$ and $K=N$ and $J \neq L$ and $J \neq K$ and (the connectives of $\Sigma)(n)$ is of type $\langle J, K\rangle \rightarrow L$ and (the connectives of $\Sigma)(n+1)$ is of type $\langle J, K, L\rangle \rightarrow J$ and (the connectives of $\Sigma)(n+2)$ is of type $\langle J\rangle \rightarrow K$ and (the connectives of $\Sigma)(n+3)$ is of type $\langle K, L\rangle \rightarrow J$.

Next we state the proposition

(71) Let $\Sigma_{1}, \Sigma_{2}$ be non empty non void connectives signatures. Suppose the connectives signature of $\Sigma_{1}=$ the connectives signature of $\Sigma_{2}$. Let $I, N$ be sets and $n$ be a natural number such that $\Sigma_{1}$ has arrays of type $I$ with connectives from $n$ and integers at $N$. Then $\Sigma_{2}$ has arrays of type $I$ with connectives from $n$ and integers at $N$.

Let $\Sigma$ be a non empty non void connectives signature, let $I, N$ be sets, let $n$ be a natural number, and let $\mathfrak{A}$ be an algebra over $\Sigma$ with defined elements. We say that $\mathfrak{A}$ has arrays of type $I$ with connectives from $n$ and integers at $N$ if and only if the condition (Def. 61) is satisfied.

(Def. 61) There exist elements $J, K$ of $\Sigma$ such that

(i) $K=I$,

(ii) (the connectives of $\Sigma)(n)$ is of type $\langle J, N\rangle \rightarrow K$,

(iii) (the defined sorts of $\mathfrak{A})(J)=($ the defined sorts of $\mathfrak{A})(K)^{\omega}$,

(iv) (the defined sorts of $\mathfrak{A})(N)=\mathbb{Z}$,

(v) for every 0-based finite array a of (the defined sorts of $\mathfrak{A})(K)$ holds for every integer $i$ such that $i \in \operatorname{dom} a$ holds (Den((the connectives of $\left.\left.\Sigma)_{n}, \mathfrak{A}\right)\right)(\langle a, i\rangle)=a(i)$ and for every defined element $x$ of $\mathfrak{A}$ from $K$ holds $\left(\operatorname{Den}\left((\text { the connectives of } \Sigma)_{n+1}, \mathfrak{A}\right)\right)(\langle a, i, x\rangle)=a+\cdot(i, x)$ and (Den( (the connectives of $\left.\left.\Sigma)_{n+2}, \mathfrak{A}\right)\right)(\langle a\rangle)=\overline{\bar{a}}$, and

(vi) for every integer $i$ and for every defined element $x$ of $\mathfrak{A}$ from $K$ such that $i \geq 0$ holds $\left(\operatorname{Den}\left((\text { the connectives of } \Sigma)_{n+3}, \mathfrak{A}\right)\right)(\langle i, x\rangle)=i \longmapsto x$.

Let $B$ be a non empty boolean signature and let $C$ be a non empty connectives signature. The functor $B+\cdot C$ yielding a strict boolean signature is defined by the conditions (Def. 62).

(Def. 62)(i) The many sorted signature of $B+\cdot C=B+\cdot C$,

(ii) the boolean sort of $B+\cdot C=$ the boolean sort of $B$, and

(iii) the connectives of $B+\cdot C=(\text { the connectives of } B)^{\frown}$ (the connectives of $C)$.

Next we state the proposition 
(72) Let $B$ be a non empty boolean signature and $C$ be a non empty connectives signature. Then

(i) the carrier of $B+\cdot C=($ the carrier of $B) \cup($ the carrier of $C)$,

(ii) the carrier' of $B+\cdot C=($ the carrier' of $B) \cup$ (the carrier' of $C$ ),

(iii) the arity of $B+\cdot C=($ the arity of $B)+\cdot($ the arity of $C)$, and

(iv) the result sort of $B+\cdot C=$ (the result sort of $B$ ) $+\cdot$ (the result sort of $C)$.

Let $B$ be a non empty boolean signature and let $C$ be a non empty connectives signature. Note that $B+\cdot C$ is non empty.

Let $B$ be a non void non empty boolean signature and let $C$ be a non empty connectives signature. One can verify that $B+\cdot C$ is non void.

Let $n_{1}, n_{2}$ be natural numbers, let $B$ be an $n_{1}$-connectives non empty non void boolean signature, and let $C$ be an $n_{2}$-connectives non empty non void connectives signature. One can check that $B+\cdot C$ is $n_{1}+n_{2}$-connectives.

One can prove the following proposition

(73) Let $M, O$ be sets and $N, I$ be sets. Suppose $I, N \in M$. Then there exists an 4-connectives non empty non void strict connectives signature $C$ such that

(i) $\quad C$ is 1-1-connectives and has arrays of type $I$ with connectives from 1 and integers at $N$,

(ii) $M \subseteq$ the carrier of $C$,

(iii) $O$ misses the carrier' of $C$, and

(iv) (the result sort of $C)(($ the connectives of $C)(2)) \notin M$.

Let $I, N$ be sets. Note that there exists a non empty non void strict connectives signature which is 4-connectives and has arrays of type $I$ with connectives from 1 and integers at $N$.

The following propositions are true:

(74) Let $n, m$ be natural numbers. Suppose $m>0$. Let $B$ be an $n$-connectives non empty non void boolean signature, $I, N$ be sets, and $C$ be a non empty non void connectives signature. Suppose $C$ has arrays of type $I$ with connectives from $m$ and integers at $N$. Then $B+\cdot C$ has arrays of type $I$ with connectives from $n+m$ and integers at $N$.

(75) Let $m$ be a natural number. Suppose $m>0$. Let $s$ be a set, $B$ be a non empty non void boolean signature, and $C$ be a non empty non void connectives signature. Suppose that

(i) $B$ has integers with connectives from $m$ and the sort at $s$, and

(ii) the carrier' of $B$ misses the carrier' of $C$.

Then $B+\cdot C$ has integers with connectives from $m$ and the sort at $s$.

(76) Let $B$ be a boolean correct non empty non void boolean signature and $C$ be a non empty non void connectives signature. Suppose the carrier' of $B$ misses the carrier' of $C$. Then $B+\cdot C$ is boolean correct. 
Let $n$ be a natural number and let $B$ be a boolean signature. We say that $B$ is $n$-array correct if and only if:

(Def. 63) (The result sort of $B)(($ the connectives of $B)(n+1)) \neq$ the boolean sort of $B$.

Let us note that there exists a strict boolean signature which is 1-1connectives, 14-connectives, 11-array correct, boolean correct, non empty, and non void and has arrays of type 1 with connectives from 11 and integers at 1 and integers with connectives from 4 and the sort at 1 .

Let $\Sigma$ be a non empty non void boolean signature with arrays of type 1 with connectives from 11 and integers at 1 . Observe that there exists a sort symbol of $\Sigma$ which is integer.

Let $\Sigma$ be a non empty non void boolean signature with arrays of type 1 with connectives from 11 and integers at 1 . The array sort of $\Sigma$ yields a sort symbol of $\Sigma$ and is defined as follows:

(Def. 64) The array sort of $\Sigma=($ the result sort of $\Sigma)(($ the connectives of $\Sigma)(12))$.

Let $\Sigma$ be a non empty non void boolean signature with integers with connectives from 4 and the sort at 1 and arrays of type 1 with connectives from 11 and integers at 1 , let $\mathfrak{A}$ be a non-empty algebra over $\Sigma$, let $a$ be an element of (the sorts of $\mathfrak{A}$ )(the array sort of $\Sigma$ ), and let $I$ be an integer sort symbol of $\Sigma$. The functor length ${ }_{I} a$ yields an element of (the sorts of $\left.\mathfrak{A}\right)(I)$ and is defined as follows:

$\left(\right.$ Def. 65) $\operatorname{length}_{I} a=(\operatorname{Den}(($ the connectives of $\Sigma)(13)(\in$ the carrier' of $\Sigma)$, $\mathfrak{A}))(\langle a\rangle)$.

Let $i$ be an element of (the sorts of $\mathfrak{A})(I)$. The functor $a(i)$ yields an element of (the sorts of $\mathfrak{A})(I)$ and is defined by:

(Def. 66) $a(i)=(\operatorname{Den}(($ the connectives of $\Sigma)(11)(\in$ the carrier' of $\Sigma), \mathfrak{A}))(\langle a, i\rangle)$.

Let $x$ be an element of (the sorts of $\mathfrak{A})(I)$. The functor $a_{i \leftarrow x}$ yielding an element of (the sorts of $\mathfrak{A}$ )(the array sort of $\Sigma$ ) is defined as follows:

(Def. 67) $a_{i \leftarrow x}=(\operatorname{Den}(($ the connectives of $\Sigma)(12)(\in$ the carrier' of $\Sigma), \mathfrak{A}))(\langle a, i$, $x\rangle)$.

Let $\Sigma$ be a boolean correct non empty non void boolean signature, let $I, s$ be sets, let $n, m$ be natural numbers, and let $\mathfrak{A}$ be a non-empty algebra over $\Sigma$ with undefined values. We say that $\mathfrak{A}$ has index overflow undefined with $n, m$, $I$, and $s$ if and only if the condition (Def. 68) is satisfied.

(Def. 68) Let $J, K$ be sort symbols of $\Sigma$. Suppose $I=J$ and $s=K$. Let $a$ be a defined element of (the sorts of $\mathfrak{A})(K)$ and $i, x$ be defined elements of (the sorts of $\mathfrak{A})(J)$. Suppose that

(i) $\quad(\operatorname{Den}(($ the connectives of $\Sigma)(n+6)(\in$ the carrier' of $\Sigma), \mathfrak{A}))(\langle(\operatorname{Den}(($ the connectives of $\Sigma)(n)(\in$ the carrier' of $\Sigma), \mathfrak{A}))(\emptyset), i\rangle)=$ false $_{\mathfrak{A}}$, or 
(ii) $\quad(\operatorname{Den}(($ the connectives of $\Sigma)(n+6)(\in$ the carrier' of $\Sigma), \mathfrak{A}))(\langle(\operatorname{Den}(($ the connectives of $\Sigma)(m+2)(\in$ the carrier' of $\Sigma), \mathfrak{A}))(\langle a\rangle), i\rangle)=$ true $_{\mathfrak{A}}$. Then

(iii) $\quad($ Den $(($ the connectives of $\Sigma)(m)(\in$ the carrier' of $\Sigma), \mathfrak{A}))(\langle a, i\rangle)=($ the undefined map of $\mathfrak{A})(J)$, and

(iv) $(\operatorname{Den}(($ the connectives of $\Sigma)(m+1)(\in$ the carrier' of $\Sigma), \mathfrak{A}))(\langle a, i, x\rangle)=$ (the undefined map of $\mathfrak{A})(K)$.

Let $\Sigma$ be a boolean correct non empty non void boolean signature with integers with connectives from 4 and the sort at 1 and arrays of type 1 with connectives from 11 and integers at 1 and let $\mathfrak{A}$ be a non-empty algebra over $\Sigma$ with undefined values. We say that $\mathfrak{A}$ has index overflow undefined if and only if:

(Def. 69) $\mathfrak{A}$ has index overflow undefined with $4,11,1$, and the array sort of $\Sigma$.

Let $\Sigma$ be a boolean correct non empty non void boolean signature with integers with connectives from 4 and the sort at 1 and arrays of type 1 with connectives from 11 and integers at 1 and let $\mathfrak{A}$ be a non-empty algebra over $\Sigma$ with undefined values. Let us observe that $\mathfrak{A}$ has index overflow undefined if and only if the condition (Def. 70) is satisfied.

(Def. 70) Let $I$ be an integer sort symbol of $\Sigma, a$ be a defined element of the sorts of $\mathfrak{A}$ )(the array sort of $\Sigma$ ), and $i, x$ be defined elements of (the sorts

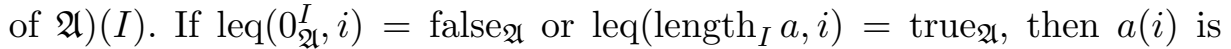
undefined and $a_{i \leftarrow x}$ is undefined.

Let $\Sigma$ be a non empty non void boolean signature with integers with connectives from 4 and the sort at 1 and arrays of type 1 with connectives from 11 and integers at 1 , let $\mathfrak{A}$ be a non-empty algebra over $\Sigma$, let $I$ be an integer sort symbol of $\Sigma$, let $i$ be an element of (the sorts of $\mathfrak{A})(I)$, and let $x$ be an element of (the sorts of $\mathfrak{A})(I)$. The functor init.array $(i, x)$ yielding an element of (the sorts of $\mathfrak{A})$ (the array sort of $\Sigma$ ) is defined as follows:

(Def. 71) init.array $(i, x)=(\operatorname{Den}(($ the connectives of $\Sigma)(14)(\in$ the carrier' of $\Sigma)$, $\mathfrak{A}))(\langle i, x\rangle)$.

Let $X$ be a non empty set. One can check that $\langle X\rangle$ is non-empty. Let $Y, Z$ be non empty sets. One can verify that $\langle X, Y, Z\rangle$ is non-empty.

Let $X$ be a functional non empty set, let $Y, Z$ be non empty sets, and let $f$ be an element of $\prod\langle X, Y, Z\rangle$. Observe that $f(1)$ is relation-like and function-like.

Let $X$ be an integer-membered non empty set, let $Y$ be a non empty set, and let $f$ be an element of $\prod\langle X, Y\rangle$. Observe that $f(1)$ is integer.

The following proposition is true

(77) Let $I, N$ be sets, $\Sigma$ be a non empty non void connectives signature with arrays of type $I$ with connectives from 1 and integers at $N, Y$ be a non empty set, and $X$ be a non-empty many sorted set indexed by $Y$. Suppose that 
(i) (the result sort of $\Sigma)(($ the connectives of $\Sigma)(2)) \notin Y$ or $X(($ the result sort of $\Sigma)(($ the connectives of $\Sigma)(2)))=X(I)^{\omega}$,

(ii) $X(N)=\mathbb{Z}$, and

(iii) $\quad I \in Y$.

Then there exists a strict algebra $\mathfrak{A}$ over $\Sigma$ with undefined values with defined elements such that

(iv) $\mathfrak{A}$ has arrays of type $I$ with connectives from 1 and integers at $N$,

(v) the defined sorts of $\mathfrak{A} \approx X$, and

(vi) for every 0-based finite array $a$ of (the defined sorts of $\mathfrak{A})(I)$ and for every integer $i$ such that $i \notin \operatorname{dom} a$ holds (Den $(($ the connectives of $\Sigma)(1)(\epsilon$ the carrier' of $\Sigma), \mathfrak{A}))(\langle a, i\rangle)=($ the undefined map of $\mathfrak{A})(I)$ and for every element $x$ of (the defined sorts of $\mathfrak{A})(I)$ holds (Den $(($ the connectives of $\Sigma)(2)(\in$ the carrier' of $\Sigma), \mathfrak{A}))(\langle a, i, x\rangle)=($ the undefined map of $\mathfrak{A})$ (the result sort of (the connectives of $\Sigma)(2)(\in$ the carrier' of $\Sigma)$ ).

Let $I, N$ be sets and let $\Sigma$ be a non empty non void connectives signature with arrays of type $I$ with connectives from 1 and integers at $N$. One can verify that there exists a strict algebra over $\Sigma$ with undefined values with defined elements which has arrays of type $I$ with connectives from 1 and integers at $N$.

Let $\Sigma_{1}$ be a non empty non void boolean signature, let $\Sigma_{2}$ be a non empty non void connectives signature, let $\mathfrak{A}_{1}$ be an algebra over $\Sigma_{1}$ with undefined values with defined elements, and let $\mathfrak{A}_{2}$ be an algebra over $\Sigma_{2}$ with undefined values with defined elements. Let us assume that the sorts of $\mathfrak{A}_{1} \approx$ the sorts of $\mathfrak{A}_{2}$ and the undefined map of $\mathfrak{A}_{1} \approx$ the undefined map of $\mathfrak{A}_{2}$. The functor $\mathfrak{A}_{1 \Sigma_{1}}+\Sigma_{\Sigma_{2}} \mathfrak{A}_{2}$ yields a strict algebra over $\Sigma_{1}+\cdot \Sigma_{2}$ with undefined values with defined elements and is defined by the conditions (Def. 72).

(Def. 72)(i) The sorts of $\mathfrak{A}_{1 \Sigma_{1}+\cdot \Sigma_{2}} \mathfrak{A}_{2}=$ (the sorts of $\left.\mathfrak{A}_{1}\right)+\cdot\left(\right.$ the sorts of $\mathfrak{A}_{2}$ ),

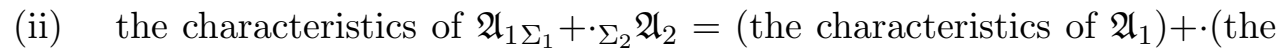
characteristics of $\mathfrak{A}_{2}$ ), and

(iii) the undefined map of $\mathfrak{A}_{1 \Sigma_{1}+\cdot \Sigma_{2}} \mathfrak{A}_{2}=$ (the undefined map of $\left.\mathfrak{A}_{1}\right)+\cdot$ (the undefined map of $\mathfrak{A}_{2}$ ).

The following propositions are true:

(78) Let $B, C$ be non empty non void connectives signatures, $\mathfrak{A}_{1}$ be an algebra over $B$ with undefined values with defined elements, and $\mathfrak{A}_{2}$ be an algebra over $C$ with undefined values with defined elements. Suppose the sorts of $\mathfrak{A}_{1} \approx$ the sorts of $\mathfrak{A}_{2}$ and the undefined map of $\mathfrak{A}_{1} \approx$ the undefined map of $\mathfrak{A}_{2}$. Then the defined sorts of $\mathfrak{A}_{1} \approx$ the defined sorts of $\mathfrak{A}_{2}$.

(79) Let $B$ be a non empty non void boolean signature, $\mathfrak{A}_{1}$ be an algebra over $B$ with undefined values with defined elements, $C$ be a non empty non void connectives signature, and $\mathfrak{A}_{2}$ be an algebra over $C$ with undefined values with defined elements. Suppose the sorts of $\mathfrak{A}_{1} \approx$ the sorts of $\mathfrak{A}_{2}$ and the undefined map of $\mathfrak{A}_{1} \approx$ the undefined map of $\mathfrak{A}_{2}$. Then the defined sorts 
of $\mathfrak{A}_{1 B}+\cdot{ }_{C} \mathfrak{A}_{2}=\left(\right.$ the defined sorts of $\left.\mathfrak{A}_{1}\right)+\cdot\left(\right.$ the defined sorts of $\left.\mathfrak{A}_{2}\right)$.

(80) Let $B$ be a boolean correct non empty non void boolean signature, $\mathfrak{A}_{1}$ be a boolean correct algebra over $B$ with undefined values with defined elements, and $C$ be a non empty non void connectives signature. Suppose the carrier' of $B$ misses the carrier' of $C$. Let $\mathfrak{A}_{2}$ be an algebra over $C$ with undefined values with defined elements. Suppose the sorts of $\mathfrak{A}_{1} \approx$ the sorts of $\mathfrak{A}_{2}$ and the undefined map of $\mathfrak{A}_{1} \approx$ the undefined map of $\mathfrak{A}_{2}$. Then $\mathfrak{A}_{1 B}+\cdot{ }_{C} \mathfrak{A}_{2}$ is boolean correct.

(81) Let $n$ be a natural number and $I$ be a set. Suppose $n \geq 4$. Let $B$ be a boolean correct non empty non void boolean signature. Suppose $B$ has integers with connectives from $n$ and the sort at $I$. Let $\mathfrak{A}_{1}$ be a boolean correct algebra over $B$ with undefined values with defined elements. Suppose $\mathfrak{A}_{1}$ has integers with connectives from $n$ and the sort at $I$. Let $C$ be a non empty non void connectives signature. Suppose the carrier' of $B$ misses the carrier' of $C$. Let $\mathfrak{A}_{2}$ be an algebra over $C$ with undefined values with defined elements. Suppose the sorts of $\mathfrak{A}_{1} \approx$ the sorts of $\mathfrak{A}_{2}$ and the undefined map of $\mathfrak{A}_{1} \approx$ the undefined map of $\mathfrak{A}_{2}$. Let $\Sigma$ be a boolean correct non empty non void boolean signature. Suppose the boolean signature of $\Sigma=B+\cdot C$. Let $\mathfrak{A}$ be a boolean correct algebra over $\Sigma$ with undefined values with defined elements. Suppose the algebra of $\mathfrak{A}$ with undefined values $=\mathfrak{A}_{1 B}+\cdot C \mathfrak{A}_{2}$. Then

(i) $\mathfrak{A}$ has integers with connectives from $n$ and the sort at $I$, and

(ii) if $\mathfrak{A}_{1}$ has division by 0 undefined with $n$ and $I$, then $\mathfrak{A}$ has division by 0 undefined with $n$ and $I$.

(82) Let $n, m$ be natural numbers and $s, r$ be sets. Suppose $n \geq 1$ and $m \geq 1$. Let $B$ be an $m$-connectives non empty non void boolean signature, $\mathfrak{A}_{1}$ be an algebra over $B$ with undefined values with defined elements, and $C$ be a non empty non void connectives signature. Suppose that

(i) the carrier' of $B$ misses the carrier' of $C$, and

(ii) $\quad C$ has arrays of type $s$ with connectives from $n$ and integers at $r$.

Let $\mathfrak{A}_{2}$ be an algebra over $C$ with undefined values with defined elements. Suppose that

(iii) the sorts of $\mathfrak{A}_{1} \approx$ the sorts of $\mathfrak{A}_{2}$,

(iv) the undefined map of $\mathfrak{A}_{1} \approx$ the undefined map of $\mathfrak{A}_{2}$, and

(v) $\mathfrak{A}_{2}$ has arrays of type $s$ with connectives from $n$ and integers at $r$.

Let $\Sigma$ be a non empty non void boolean signature. Suppose the boolean signature of $\Sigma=B+\cdot C$. Let $\mathfrak{A}$ be an algebra over $\Sigma$ with undefined values with defined elements. Suppose the algebra of $\mathfrak{A}$ with undefined values $=\mathfrak{A}_{1 B}+\cdot{ }_{C} \mathfrak{A}_{2}$. Then

(vi) $\mathfrak{A}$ has arrays of type $s$ with connectives from $m+n$ and integers at $r$, and 
(vii) if the characteristics of $\mathfrak{A}_{1} \approx$ the characteristics of $\mathfrak{A}_{2}$ and $B \approx C$ and $\mathfrak{A}_{1}$ is undefined consequently and $\mathfrak{A}_{2}$ is undefined consequently, then $\mathfrak{A}$ is undefined consequently.

(83) Let $n, n_{1}, m$ be natural numbers and $r$ be a set. Suppose $n \geq 1$ and $n_{1} \geq 4$. Let $B$ be a boolean correct non empty non void boolean signature. Suppose $B$ is $m$-connectives. Let $\mathfrak{A}_{1}$ be a boolean correct algebra over $B$ with undefined values with defined elements. Suppose that

(i) $B$ has integers with connectives from $n_{1}$ and the sort at $r$, and

(ii) $\mathfrak{A}_{1}$ has integers with connectives from $n_{1}$ and the sort at $r$.

Let $C$ be a non empty non void connectives signature. Suppose that

(iii) the carrier' of $B$ misses the carrier' of $C$, and

(iv) $C$ has arrays of type $r$ with connectives from $n$ and integers at $r$.

Let $\mathfrak{A}_{2}$ be an algebra over $C$ with undefined values with defined elements. Suppose that

(v) the sorts of $\mathfrak{A}_{1} \approx$ the sorts of $\mathfrak{A}_{2}$,

(vi) the undefined map of $\mathfrak{A}_{1} \approx$ the undefined map of $\mathfrak{A}_{2}$, and

(vii) $\mathfrak{A}_{2}$ has arrays of type $r$ with connectives from $n$ and integers at $r$.

Let $\Sigma$ be a boolean correct non empty non void boolean signature. Suppose the boolean signature of $\Sigma=B+\cdot C$. Let $A$ be a boolean correct algebra over $\Sigma$ with undefined values with defined elements such that the algebra of $\mathfrak{A}$ with undefined values $=\mathfrak{A}_{1 B}+\cdot{ }_{C} \mathfrak{A}_{2}$ and for every 0-based finite array $a$ of $\mathbb{Z}$ and for every integer $i$ such that $i \notin \operatorname{dom} a$ holds (Den $\left((\right.$ the connectives of $C)(n)(\in$ the carrier' of $\left.\left.C), \mathfrak{A}_{2}\right)\right)(\langle a, i\rangle)=$ (the undefined map of $\left.\mathfrak{A}_{2}\right)(r)$ and for every integer $x$ holds (Den((the connectives of $C)(n+1)(\in$ the carrier' of $\left.\left.C), \mathfrak{A}_{2}\right)\right)(\langle a, i, x\rangle)=($ the undefined map of $\mathfrak{A}_{2}$ ) (the result sort of (the connectives of $\left.C\right)(n+1)(\in$ the carrier' of $C)$ ). Then $\mathfrak{A}$ has index overflow undefined with $n_{1}, n+m, r$, and the result sort of the connectives of $\Sigma(n+m+1)(\in$ the carrier' of $\Sigma)$.

(84) Let $n$ be a natural number, $s$ be a set, and $\Sigma_{1}, \Sigma_{2}$ be boolean signatures. Suppose that

(i) the boolean sort of $\Sigma_{1}=$ the boolean sort of $\Sigma_{2}$,

(ii) len (the connectives of $\left.\Sigma_{2}\right) \geq 3$, and

(iii) for every $i$ such that $i \geq 1$ and $i \leq 3$ holds (the arity of $\left.\Sigma_{1}\right)(($ the connectives of $\left.\left.\Sigma_{1}\right)(i)\right)=\left(\right.$ the arity of $\left.\Sigma_{2}\right)\left(\left(\right.\right.$ the connectives of $\left.\left.\Sigma_{2}\right)(i)\right)$ and (the result sort of $\left.\Sigma_{1}\right)\left(\left(\right.\right.$ the connectives of $\left.\left.\Sigma_{1}\right)(i)\right)=($ the result sort of $\left.\Sigma_{2}\right)\left(\left(\right.\right.$ the connectives of $\left.\left.\Sigma_{2}\right)(i)\right)$.

If $\Sigma_{1}$ is boolean correct, then $\Sigma_{2}$ is boolean correct.

(85) Let $n$ be a natural number, $s$ be a set, and $\Sigma_{1}, \Sigma_{2}$ be non empty boolean signatures. Suppose that $n \geq 1$ and the boolean sort of $\Sigma_{1}=$ the boolean sort of $\Sigma_{2}$ and len (the connectives of $\left.\Sigma_{2}\right) \geq n+6$ and (the connectives of $\left.\Sigma_{2}\right)(n) \neq\left(\right.$ the connectives of $\left.\Sigma_{2}\right)(n+1)$ and (the connecti- 
ves of $\left.\Sigma_{2}\right)(n+3) \neq\left(\right.$ the connectives of $\left.\Sigma_{2}\right)(n+4)$ and (the connectives of $\left.\Sigma_{2}\right)(n+3) \neq$ (the connectives of $\left.\Sigma_{2}\right)(n+5)$ and (the connectives of $\left.\Sigma_{2}\right)(n+4) \neq\left(\right.$ the connectives of $\left.\Sigma_{2}\right)(n+5)$ and for every $i$ such that $i \geq n$ and $i \leq n+6$ holds (the arity of $\left.\Sigma_{1}\right)\left(\left(\right.\right.$ the connectives of $\left.\left.\Sigma_{1}\right)(i)\right)=($ the arity of $\left.\Sigma_{2}\right)\left(\left(\right.\right.$ the connectives of $\left.\left.\Sigma_{2}\right)(i)\right)$ and (the result sort of $\left.\Sigma_{1}\right)(($ the connectives of $\left.\left.\Sigma_{1}\right)(i)\right)=\left(\right.$ the result sort of $\left.\Sigma_{2}\right)\left(\left(\right.\right.$ the connectives of $\left.\left.\Sigma_{2}\right)(i)\right)$. Suppose $\Sigma_{1}$ has integers with connectives from $n$ and the sort at $s$. Then $\Sigma_{2}$ has integers with connectives from $n$ and the sort at $s$.

(86) Let $n, m$ be natural numbers, $s, r$ be sets, and $\Sigma_{1}, \Sigma_{2}$ be non empty connectives signatures. Suppose that

(i) $1 \leq n$,

(ii) len (the connectives of $\left.\Sigma_{1}\right) \geq n+3$, and

(iii) for every $i$ such that $i \geq n$ and $i \leq n+3$ holds (the arity of $\left.\Sigma_{1}\right)(($ the connectives of $\left.\left.\Sigma_{1}\right)(i)\right)=\left(\right.$ the arity of $\left.\Sigma_{2}\right)\left(\left(\right.\right.$ the connectives of $\left.\left.\Sigma_{2}\right)(i+m)\right)$ and (the result sort of $\left.\Sigma_{1}\right)\left(\left(\right.\right.$ the connectives of $\left.\left.\Sigma_{1}\right)(i)\right)=$ (the result sort of $\left.\Sigma_{2}\right)\left(\left(\right.\right.$ the connectives of $\left.\left.\Sigma_{2}\right)(i+m)\right)$.

Suppose $\Sigma_{2}$ has arrays of type $s$ with connectives from $n+m$ and integers at $r$. Then $\Sigma_{1}$ has arrays of type $s$ with connectives from $n$ and integers at $r$.

(87) Let $j, k$ be sets and $i, m, n$ be natural numbers. Suppose $m \geq 4$ and $m+6 \leq n$ and $i \geq 1$. Let $\Sigma$ be a 1-1-connectives boolean correct non empty non void boolean signature. Suppose that

then there exists a boolean correct non empty non void boolean signature $B$ and there exists a non empty non void connectives signature $C$ such that

the boolean signature of $\Sigma=B+\cdot C$ and $B$ is $n$-connectives and has integers with connectives from $m$ and the sort at $k$ and $C$ has arrays of type $j$ with connectives from $i$ and integers at $k$ and the carrier of $B=$ the carrier of $C$ and the carrier' of $B=$ (the carrier' of $\Sigma) \backslash \mathrm{rng}$ (the connectives of $C$ ) and the carrier' of $C=\operatorname{rng}$ (the connectives of $C$ ) and the connectives of $B=($ the connectives of $\Sigma)\lceil n$ and the connectives of $C=$ (the connectives of $\Sigma)_{\lfloor n}$.

(88) Let $s, I$ be sets and $\Sigma$ be a boolean correct non empty non void boolean signature. Suppose $\Sigma$ has integers with connectives from 4 and the sort at $I$. Let $X$ be a non empty set. Suppose $s \in$ the carrier of $\Sigma$ and $s \neq I$ and $s \neq$ the boolean sort of $\Sigma$. Then there exists a boolean correct strict algebra $\mathfrak{A}$ over $\Sigma$ with undefined values with defined elements such that

(i) the undefined map of $\mathfrak{A}=$ the defined sorts of $\mathfrak{A}$,

(ii) the sorts of $\mathfrak{A}=\operatorname{succ}$ (the defined sorts of $\mathfrak{A}$ ),

(iii) $\mathfrak{A}$ is undefined consequently and has integers with connectives from 4 and the sort at $I$, 
(iv) (the defined sorts of $\mathfrak{A})(s)=X$, and

(v) $\mathfrak{A}$ has division by 0 undefined with 4 and $I$.

Let $\Sigma$ be a 1-1-connectives 11-array correct boolean correct non empty non void boolean signature with arrays of type 1 with connectives from 11 and integers at 1 and integers with connectives from 4 and the sort at 1 . One can check that there exists a boolean correct strict algebra over $\Sigma$ with undefined values with defined elements which is undefined consequently and has arrays of type 1 with connectives from 11 and integers at 1 , integers with connectives from 4 and the sort at 1 , division by 0 undefined, and index overflow undefined.

\section{REFERENCES}

[1] Grzegorz Bancerek. Cardinal numbers. Formalized Mathematics, 1(2):377-382, 1990.

[2] Grzegorz Bancerek. Introduction to trees. Formalized Mathematics, 1(2):421-427, 1990.

[3] Grzegorz Bancerek. König's theorem. Formalized Mathematics, 1(3):589-593, 1990.

[4] Grzegorz Bancerek. The ordinal numbers. Formalized Mathematics, 1(1):91-96, 1990.

[5] Grzegorz Bancerek. Cartesian product of functions. Formalized Mathematics, 2(4):547552,1991

[6] Grzegorz Bancerek. König's lemma. Formalized Mathematics, 2(3):397-402, 1991.

[7] Grzegorz Bancerek. Algebra of morphisms. Formalized Mathematics, 6(2):303-310, 1997.

[8] Grzegorz Bancerek. Institution of many sorted algebras. Part I: Signature reduct of an algebra. Formalized Mathematics, 6(2):279-287, 1997.

[9] Grzegorz Bancerek. Mizar analysis of algorithms: Preliminaries. Formalized Mathematics, 15(3):87-110, 2007, doi:10.2478/v10037-007-0011-x.

[10] Grzegorz Bancerek. Sorting by exchanging. Formalized Mathematics, 19(2):93-102, 2011, doi: 10.2478/v10037-011-0015-4.

[11] Grzegorz Bancerek. Free term algebras. Formalized Mathematics, 20(3):239-256, 2012, doi: 10.2478/v10037-012-0029-6.

[12] Grzegorz Bancerek and Krzysztof Hryniewiecki. Segments of natural numbers and finite sequences. Formalized Mathematics, 1(1):107-114, 1990.

[13] Grzegorz Bancerek and Piotr Rudnicki. The set of primitive recursive functions. Formalized Mathematics, 9(4):705-720, 2001.

[14] Grzegorz Bancerek and Andrzej Trybulec. Miscellaneous facts about functions. Formalized Mathematics, 5(4):485-492, 1996.

[15] Ewa Burakowska. Subalgebras of the universal algebra. Lattices of subalgebras. Formalized Mathematics, 4(1):23-27, 1993.

[16] Czesław Byliński. Binary operations. Formalized Mathematics, 1(1):175-180, 1990.

[17] Czesław Byliński. Finite sequences and tuples of elements of a non-empty sets. Formalized Mathematics, 1(3):529-536, 1990.

[18] Czesław Byliński. Functions and their basic properties. Formalized Mathematics, 1(1):5565, 1990.

[19] Czesław Byliński. Functions from a set to a set. Formalized Mathematics, 1(1):153-164, 1990.

[20] Czesław Bylinski. The modification of a function by a function and the iteration of the composition of a function. Formalized Mathematics, 1(3):521-527, 1990.

[21] Czesław Byliński. Partial functions. Formalized Mathematics, 1(2):357-367, 1990.

[22] Czesław Byliński. Some basic properties of sets. Formalized Mathematics, 1(1):47-53, 1990.

[23] Agata Darmochwał. Finite sets. Formalized Mathematics, 1(1):165-167, 1990.

[24] Artur Korniłowicz. On the group of automorphisms of universal algebra \& many sorted algebra. Formalized Mathematics, 5(2):221-226, 1996.

[25] Artur Korniłowicz and Marco Riccardi. The Borsuk-Ulam theorem. Formalized Mathematics, 20(2):105-112, 2012, doi: 10.2478/v10037-012-0014-0.

[26] Małgorzata Korolkiewicz. Homomorphisms of many sorted algebras. Formalized Mathematics, 5(1):61-65, 1996. 
[27] Jarosław Kotowicz, Beata Madras, and Małgorzata Korolkiewicz. Basic notation of universal algebra. Formalized Mathematics, 3(2):251-253, 1992.

[28] Yatsuka Nakamura and Grzegorz Bancerek. Combining of circuits. Formalized Mathematics, 5(2):283-295, 1996.

[29] Andrzej Nedzusiak. Probability. Formalized Mathematics, 1(4):745-749, 1990.

[30] Beata Perkowska. Free many sorted universal algebra. Formalized Mathematics, 5(1):6774, 1996.

[31] Andrzej Trybulec. Binary operations applied to functions. Formalized Mathematics, 1(2):329-334, 1990.

[32] Andrzej Trybulec. Enumerated sets. Formalized Mathematics, 1(1):25-34, 1990.

[33] Andrzej Trybulec. Many sorted sets. Formalized Mathematics, 4(1):15-22, 1993.

[34] Andrzej Trybulec. Many sorted algebras. Formalized Mathematics, 5(1):37-42, 1996.

[35] Andrzej Trybulec. A scheme for extensions of homomorphisms of many sorted algebras. Formalized Mathematics, 5(2):205-209, 1996.

[36] Andrzej Trybulec. On the sets inhabited by numbers. Formalized Mathematics, 11(4):341$347,2003$.

[37] Michał J. Trybulec. Integers. Formalized Mathematics, 1(3):501-505, 1990.

[38] Wojciech A. Trybulec. Pigeon hole principle. Formalized Mathematics, 1(3):575-579, 1990.

[39] Zinaida Trybulec. Properties of subsets. Formalized Mathematics, 1(1):67-71, 1990

[40] Tetsuya Tsunetou, Grzegorz Bancerek, and Yatsuka Nakamura. Zero-based finite sequences. Formalized Mathematics, 9(4):825-829, 2001.

[41] Edmund Woronowicz. Many argument relations. Formalized Mathematics, 1(4):733-737, 1990.

[42] Edmund Woronowicz. Relations and their basic properties. Formalized Mathematics, 1(1):73-83, 1990.

Received August 27, 2012 